\title{
Cryo-EM structures reveal transcription initiation steps by yeast mitochondrial RNA polymerase
}

Brent De Wijngaert ${ }^{1}$, Shemaila Sultana ${ }^{2}$, Anupam Singh ${ }^{2}$, Chhaya Dharia ${ }^{2}$, Hans Vanbuel $^{1}$, Jiayu Shen ${ }^{2}$, Daniel Vasilchuk ${ }^{2}$, Sergio E. Martinez ${ }^{1}$, Eaazhisai Kandiah ${ }^{3}$, Smita S. Patel ${ }^{2, *}$, Kalyan Das ${ }^{1,4,{ }^{*}}$

\section{Affiliations:}

${ }^{1}$ Rega Institute for Medical Research, The Department of Microbiology, Immunology and Transplantation, KU Leuven, 3000 Leuven, Belgium

${ }^{2}$ Department of Biochemistry and Molecular Biology, Robert Wood Johnson Medical School, Rutgers University, Piscataway, NJ 08854, USA

${ }^{3}$ European Synchrotron Radiation Facility, 71, avenue des Martyrs, 38043 Grenoble, France

${ }^{4}$ Lead Contact

*Correspondence: kalyan.das@kuleuven.be and patelss@rwims.rutgers.edu 


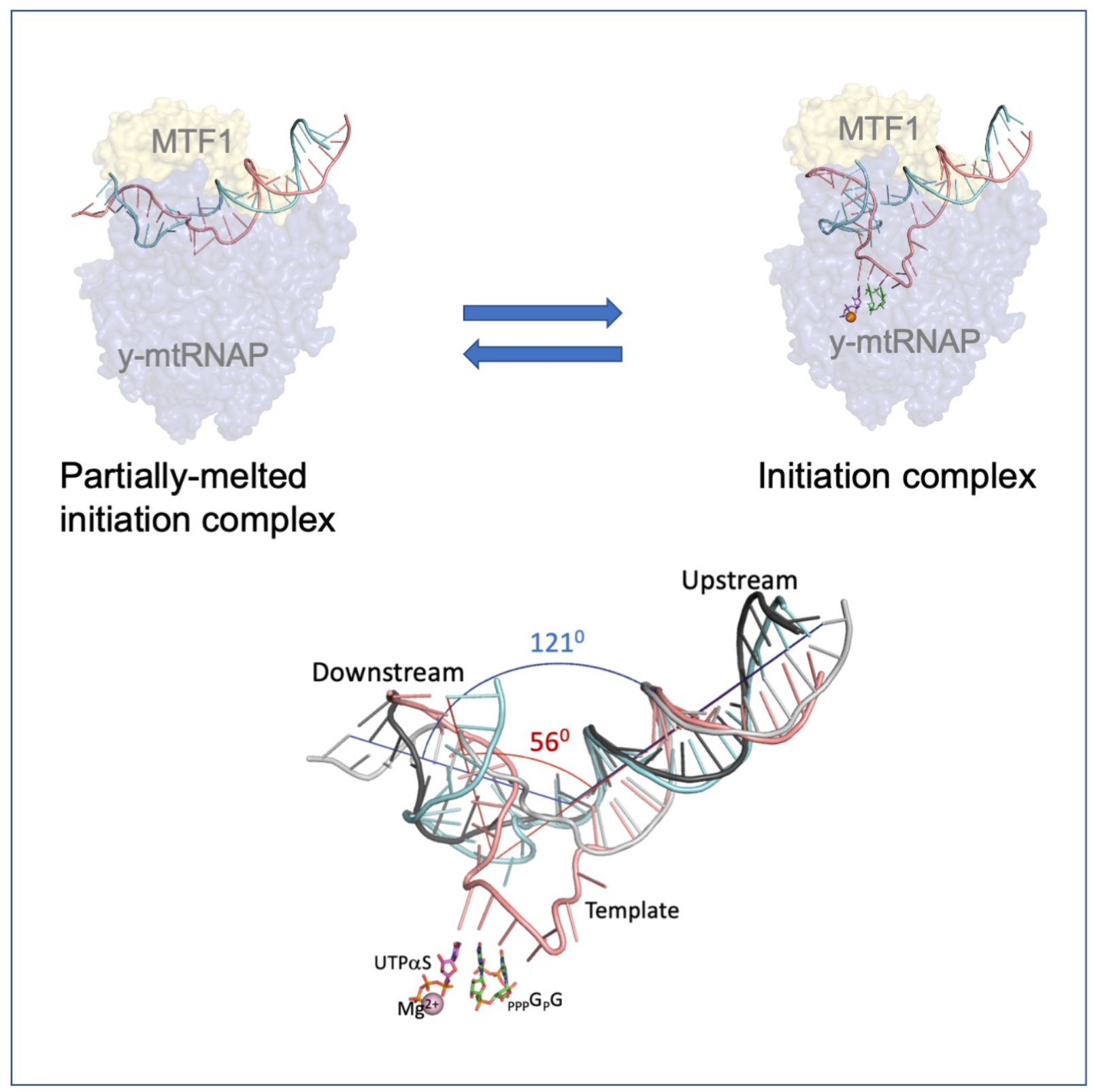




\section{SUMMARY}

Mitochondrial RNA polymerase (mtRNAP) is crucial in cellular energy production yet, understanding of mitochondrial DNA transcription initiation lags that of bacterial and nuclear DNA transcription. We report structures of two transcription initiation intermediate states of yeast mtRNAP that explain promoter melting, template alignment, DNA scrunching, abortive synthesis, and transition into elongation. In partially-melted initiation complex (PmIC), transcription factor MTF1 makes base-specific interactions with flipped non-template (NT) -4 to -1 nucleotides 'AAGT' of the DNA promoter. In the initiation complex (IC), the template in the expanded 7-mer bubble positions the RNA and NTP analog, UTP $\alpha$ S, while NT scrunches into an NT-loop. The scrunched NT-loop is stabilized by centrally positioned MTF1 C-tail. The IC and PmIC states coexist in solution revealing a dynamic equilibrium between two functional states. Frequent scrunching/unscruching transitions, and the imminent steric clashes of inflating NT-loop and growing RNA:DNA with the C-tail explain abortive synthesis and transition into elongation.

\section{KEYWORDS}

Mitochondria; RNA polymerase; transcription factor; MTF1; TFB2M; RPO41; POLRMT; RNAP 


\section{INTRODUCTION}

Cellular DNAs are transcribed into RNAs by DNA-dependent RNA polymerases (RNAPs) in a regulated multi-step process. The bacterial and eukaryotic nuclear genomes are transcribed by multi-subunit RNAPs that typically depend on one or more transcription factors for promoter-specific transcription initiation (Browning and Busby, 2004; Cramer, 2019). In contrast, mitochondrial DNAs in eukaryotes are transcribed by single-subunit RNAPs that are related to T-odd lineage of bacteriophage RNAPs. Unlike phage T7 RNAP, which does not require any transcription factors (Cheetham and Steitz, 2000), mitochondrial RNAPs (mtRNAPs) depend on accessory transcription factors for promoter-specific initiation, similar to the multi-subunit RNAPs (Gustafsson et al., 2016; Hillen et al., 2018). Despite differences in details, all RNAPs catalyze transcription initiation by a standard sequence of events consisting of (i) promoter-specific recognition, (ii) formation of a transcription initiation bubble, (iii) DNA scrunching and synthesis of short RNA products, and finally (iv) promoter release and transition from initiation to elongation phase.

The mitochondrial DNA codes for essential proteins of the respiratory complex which synthesizes ATP. Despite the importance of mitochondrial DNA transcription in energy production and human health, we lack a basic understanding of the mechanism of transcription by mtRNAPs. Much of our understanding of mtRNAPs comes from studies of human and yeast (Saccharomyces cerevisiae) mtRNAPs (Deshpande and Patel, 2012; Gustafsson et al., 2016; Hillen et al., 2018). The yeast system has served as a model system, both genetically and biochemically, for understanding the transcription machinery of mitochondria. The yeast mtRNAP initiation complex (y-mtRNAP IC) is a two-component system consisting of the catalytic subunit y-mtRNAP (also referred as RPO41) and transcription factor MTF1 (Kim et al., 2012; Matsunaga and Jaehning, 2004; McCulloch et al., 2002; Tang et al., 2011). The human mtRNAP initiation complex (h-mtRNAP IC) contains three protein components - the catalytic subunit h-mtRNAP (also referred as POLRMT) and two transcription factors, TFAM and TFB2M (Falkenberg et al., 2002; Fisher et al., 1992; Posse and Gustafsson, 2017; Ramachandran et al., 2017). The yeast mtDNA promoters contain a nine-nucleotide conserved sequence (-8) ATATAAGTA(+1) (Biswas et al., 1987; Osinga et al., 1982) in contrast, the human promoters are more diverse. MTF1 and TFB2M 
are structurally and functionally homologous, and are essential for promoter melting. Both MTF1 and TFB2M drive promoter melting by trapping the non-template (NT) strand of the transcription bubble (Hillen et al., 2017; Paratkar and Patel, 2010; Tang et al., 2009). Promoter melting is a crucial step for transcription initiation, and it is well-studied in multisubunit RNAPs. For example, a recent cryo-EM study of bacterial RNAP has captured structures of intermediate states leading from a closed to an open bubble complex in the presence of the transcription factor TraR (Chen et al., 2020). Analogous intermediate states between closed and open complexes are not identified in T7 RNAP or mtRNAPs. The structure of the h-mtRNAP IC was captured in an inactive fingers-clenched state with a significant part of the transcription bubble disordered (Hillen et al., 2017). Hence, the structural basis for promoter melting remains mostly unknown for the mtRNAPs.

Open complex formation is followed by the synthesis of short RNA transcripts from 2- to 8mer in length. The downstream DNA must unwind to provide the template for RNA synthesis. However, the upstream promoter DNA remains stably bound to the RNAP throughout initiation; therefore, the transcription bubble progressively gets larger with RNA synthesis. The expanding bubble is accommodated in the active-site cavity through DNA scrunching, which is a general mechanism observed in both multi- and single-subunit RNAPs (Kapanidis et al., 2006; Tang et al., 2008) including y-mtRNAP (Sohn et al., 2020). Despite its widespread acceptance as a mechanism for transcription initiation, the scrunched DNA conformation has not been visualized in any system. Recent biochemical studies have identified the elements in y-mtRNAP, such as the C-terminal tail (C-tail) of MTF1 that stabilize the scrunched DNA conformation in the initiation complex (Basu et al., 2020). However, the structural basis for DNA scrunching is not understood.

Understanding the mechanism of transcription initiation requires capturing key intermediate states and characterizing them biochemically and structurally. The related T7 RNAP has been extensively characterized through structural studies; however, the forked promoters used in those studies lacked a complete transcription bubble and therefore, the structures were not ideal for capturing the promoter melting or the DNA scrunching events (Cheetham and Steitz, 1999, 2000). In general, the mechanism of promoter melting and DNA scrunching in single-subunit RNAPs remains elusive. Additionally, the absence of structures 
of mtRNAPs from lower eukaryotes leaves a gap in understanding the conserved and nonconserved features across single-stranded RNAPs.

Here we report single-particle cryo-EM structure of a previously undiscovered partiallymelted transcription initiation complex (PmIC) of y-mtRNAP:MTF1:promoter DNA, and the structure of the transcription initiation complex (IC) of y-mtRNAP:MTF1:promoter DNA:2mer RNA:UTP $\alpha$ S at 3.1 and $3.7 \AA$ resolution, respectively. The structures reveal how (i) the MTF1 recognizes a stretch of four nucleotides "(-4)AAGT(-1)" in the NT strand and opens the promoter from -4 to -1 to initiate transcription bubble formation in the PmIC state, (ii) the transition from PmIC to IC is accomplished by expansion of the transcription bubble and alignment of the template with RNA and NTP at the active site, (iii) the NT strand is scrunched during RNA synthesis, and (iv) the MTF1 C-tail stabilizes the scrunched DNA. Visualization of the transcription bubble in IC helps understand how the scrunched NT strand and the growing RNA:DNA hybrid duplex is involved in triggering conformational changes required for the transition from transcription initiation to the elongation phase.

\section{RESULTS AND DISCUSSION}

\section{The PmIC and IC coexist during transcription initiation}

For structural studies, y-mtRNAP $(\Delta \mathrm{N} 100)$ and MTF1 proteins were assembled on a premelted promoter DNA ( -21 to +12 region of 15 S yeast mtDNA promoter; Figure S1A). We used $\Delta$ N100 y-mtRNAP because it provided a better particle distribution on cryo-EM grids at the initial screening stage. Moreover, previous studies have shown that deletion of $100 \mathrm{~N}$ terminal residues in $\triangle \mathrm{N} 100 \mathrm{y}$-mtRNAP has minimal effect on transcription initiation (Paratkar et al., 2011). Studies have shown that a promoter DNA melts from positions -4 to +2 during initiation complex formation (Tang et al., 2009). Therefore, we introduced mismatches in the -4 to +2 region to create a pre-melted initiation bubble. We only mutated the template strand because MTF1 appears to recognize the NT strand in a sequence-specific manner (Paratkar and Patel, 2010). The complex of y-mtRNAP, MTF1, and the promoter DNA was purified by size-exclusion chromatography. Light scattering and thermal-shift experiments ascertained that the complex was stable and homogeneous for cryo-EM studies (Figure S1B-F). Experimental cryo-EM map, as discussed later, revealed that this sample 
generated a partially melted initiation complex (y-mtRNAP PmIC). To prepare y-mtRNAP initiation complex (y-mtRNAP IC), we incubated the y-mtRNAP PmIC sample with a 2-mer RNA, pppGpG, and an NTP analog, UTP $\alpha$ S, that are complementary to the +1 to +3 template sequence. Interestingly, single-particle cryo-EM data analysis of y-mtRNAP IC (Figure S2) revealed the presence of both IC and PmIC states in the y-mtRNAP IC sample despite the addition of a six-fold molar excess RNA and 50-fold molar excess UTP $\alpha \mathrm{S}$ - the condition that was expected to shift the equilibrium to IC completely. The co-existence of PmIC and IC states suggests a dynamic equilibrium between the two states. A recent single-molecule FRET study of transcription initiation has observed dynamic equilibrium among intermediate IC states of y-mtRNAP (Sohn et al., 2020). From the cryo-EM dataset, the final density maps for y-mtRNAP IC and y-mtRNAP PmIC structures were calculated at $3.7 \AA$ and $3.5 \AA ̊$ resolution, respectively (Table 1). To ascertain the presence of a stable PmIC state, we prepared cryoEM grids of the purified complex from the gel-filtration sample without adding RNA or UTP. The new density map confirmed the structural state of PmIC and extended the resolution to $3.1 \AA$. The steps of transcription initiation discussed in this paper are derived from the analysis of y-mtRNAP PmIC and IC structures at $3.1 \AA$ and $3.7 \AA ̊$ resolution, respectively.

\section{Structure of $y$-mtRNAP}

The 3.1 A EM density map generated a reliable 3D structure of the $y$-mtRNAP subunit. Figure 1A schematically defines the shared structural elements in y-mtRNAP, h-mtRNAP, and T7 RNAP that are expected to perform similar functions. The y-mtRNAP has a characteristic right-hand shaped C-terminal domain (CTD) with palm, fingers, and thumb subdomains (Figure 1B). The palm contains the polymerase active site which is responsible for RNA synthesis. Single-subunit RNAPs and A-family DNA polymerases share a common active site and NTP binding pocket that performs the nucleotide addition reaction facilitated by two $\mathrm{Mg}^{2+}$-ion dependent catalytic mechanism (Steitz, 1999). About 800 amino acid residues in the CTD of y-mtRNAP have $28 \%$ and $41 \%$ sequence identity with the CTDs of T7 RNAP and h-mtRNAP, respectively (Figure S3A). Interestingly, the CTD of y-mtRNAP contains a stretch of about 100 amino acid residues from 1232 to 1328, which is not present in T7 RNAP or hmtRNAP. We have partially traced this non-conserved subdomain, which interacts with the 
downstream DNA in the y-mtRNAP IC structure suggesting that this subdomain may be involved in stabilizing the bent DNA conformation.

The N-terminal domain (NTD) of the RNAPs contains some of the promoter-binding elements (Figure S3B). Despite the low sequence similarity between the NTD of y-mtRNAP and h-mtRNAP, helices $\alpha \mathrm{D}$ to $\alpha$ I that form the promoter-binding domain (PBD) in the NTDs of y-mtRNAP and h-mtRNAP align structurally and show common structural features, such as the AT-rich recognizing loop and intercalating hairpin (Figure S3C) (Cheetham and Steitz, 1999). In $y$-mtRNAP, the AT-rich recognizing loop has less extensive interactions with the promoter compared to that of T7 RNAP. The h-mtRNAP IC relies significantly less on the ATrich recognizing loop for promoter binding, most likely because TFAM provides additional upstream DNA contacts. Additionally, the N-terminal extension (NTE) of h-mtRNAP has a penta-tricopeptide repeat (PPR) domain, which contacts the -10 to -15 promoter region (Hillen et al., 2017). y-mtRNAP also contains an NTE region, which is absent in the T7 RNAP (Figure 1A). Analysis of y-mtRNAP sequence predicts the presence of PPR domain in $y$ mtRNAP (Lipinski et al., 2011). However, the NTE region is disordered and y-mtRNAP is traced from the NTD residue 385. Hence, we cannot confirm the presence of the PPR domain in $\mathrm{y}$-mtRNAP. Nevertheless, previous biochemical studies have shown that $\Delta \mathrm{N} 380 \mathrm{y}-$ mtRNAP, with $380 \mathrm{~N}$-terminal residues deleted, is defective in transcription initiation on duplex promoter but competent on a pre-melted promoter suggesting that at least a part of the NTE is necessary for promoter melting (Paratkar et al., 2011).

The other key promoter binding structural elements, the intercalating hairpin (ICH) and specificity loop (SL) are resolved well in y-mtRNAP structure (Figure S4A). In T7 RNAP, the specificity loop is involved in promoter recognition, wherein, the residues R647, R758, and Q756 form base-specific interactions with -7, -8, and -9 template bases. The specificity loop in $y$-mtRNAP is positioned at the same location on the promoter DNA as in T7 RNAP. Several residues of y-mtRNAP specificity loop (K1127, Q1129, Q1135, and T1136 ) that were biochemically shown to be critical for promoter recognition (Nayak et al., 2009) are found in our structure to interact predominantly with the sugar-phosphate backbone of the template strand, except for Q1129 (Figure S4B). The residue Q1129 makes base-specific H-bond 
interactions, and T1136 side chain forms H-bond with phosphate oxygen of the -7 template nucleotide, respectively. Residue K1127 has H-bond interactions with the phosphate of the 4 template nucleotide, and Q1135 interacts with the phosphate of the -6 template nucleotide. Additionally, the phosphates of -6 and -7 template nucleotides are involved in $\mathrm{H}$-bond interactions with the main chain amino groups of F1138 and T1136, respectively. We conclude that the specificity loop in y-mtRNAP is involved in promoter recognition, albeit to a lesser extent than in T7 RNAP. Transcription initiation by T7 RNAP is not facilitated by a transcription factor; hence, T7 RNAP relies more heavily on base-specific interactions with the promoter for initiation complex formation. T7 promoters contain a longer promoterrecognition sequence from -17 to +2 (Imburgio et al., 2000) as compared to a shorter nine nucleotide $\mathrm{y}$-mt promoter-recognition site. The specificity loop is partly ordered in hmtRNAP IC structure (Hillen et al., 2017) suggesting that the h-mtRNAP IC may be less dependent on the specificity loop.

\section{Interactions between $y$-mtRNAP and MTF1}

The overall architecture of MTF1:y-mtRNAP complex is analogous to TFB2M:h-mtRNAP. There are multiple points of contact between MTF1 and y-mtRNAP. A crescent-shaped platform in y-mtRNAP carved by a hairpin (residues 613 - 633) on one side and the intercalating hairpin on the other side supports the CTD (residues 252-325) of MTF1 in the PmIC and IC states (Figure S4C-D). This structural element (residues 613 - 633) is absent in T7 RNAP and disordered in h-mtRNAP IC structure (Hillen et al., 2017). However, biochemical studies of h-mtRNAP are consistent with the role of this hairpin in supporting TFB2M (Morozov et al., 2015). Based on its structure and specific role in supporting the initiation factor (Figure S4E), we define the hairpin (residues 613 - 633) as MTF1/B2 hairpin (also referred as B2 loop). The region in MTF1 (residues 321-325) that interacts with the MTF1/B2 hairpin is highly conserved in yeast and fungi species (Figure S5A). Another point of contact between MTF1 and y-mtRNAP is through the thumb subdomain. The NTD (residues 2-251) of MTF1 interacts with the tip of the thumb helix (Figure S4D). The C153 R162 region in MTF1 interact with the thumb and is conserved in a wide range of fungi species (Figure S5A); the residues N158 and W159 are strictly conserved, K/R/Q157 is polar, and R/K162 is positively charged. Biochemically, these thumb subdomain interactions 
promote stable binding of MTF1 to y-mtRNAP (Velazquez et al., 2015). Herein, we show that mutating the MTF1 residues that interact with the thumb also weakens the interactions between y-mtRNAP and MTF1 (Figures S4F-G).

\section{Promoter DNA is partially melted in the PmIC state}

We discovered PmIC as a new intermediate state in the initiation pathway that contains a stable transcription core composed of y-mtRNAP, MTF1, and promoter DNA from -18 to +8 (Figures 2A-C). The PmIC structure reveals how MTF1 and y-mtRNAP initiate promoter melting by unwinding the -4 to -1 DNA region and creating a 4-nucleotide transcription bubble. Although we provided a pre-melted promoter with six mismatches from position -4 to +2 , the +1 and +2 nucleotides assumed a duplex-like DNA conformation in PmIC, albeit lacking canonical base pairing. The structure reveals that sequence-specific interactions of MTF1 with the promoter NT strand in the bubble are crucial in initiating DNA melting. MTF1 shows base-specific interactions with NT nucleotides -3 to -1 of the nine-nucleotide promoter recognition site. Conserved bases of (-4)AAGT(-1) flip into a cleft formed by the residues 103-105, 144-148, and 190-192 at the interface of the $\mathrm{N}$ - and C-terminal domains of MTF1. We term this cleft as the "NT groove" (Figure 2D). The flipped -1 thymine base stacks with Y103. The -2 guanine base is sandwiched between the aromatic side chains of Y103 and W105, and all $\mathrm{N}$ and $\mathrm{O}$ atoms of the -2 base, except N7, are engaged in H-bond interactions with the main chain carbonyl of Y103, main-chain amino group of W105, and the side chain of Q149 (Figure 2E; stereo view in Figure S4H). The MTF1 residues, W105 and Q149, are strictly conserved in fungi MTF1, and Y103 is substituted only with F/H preserving the aromaticity (Figure S5A). This network of interactions explains why guanine at -2 NT position is essential, and its substitution severely impairs promoter melting and transcription initiation (Biswas and Getz, 1986; Tang et al., 2011). The -3 and -4 AA bases are stacked together and sandwiched by the intercalating hairpin on one side and an MTF1 helix $\alpha 6$ (residues 144-156) on the other side (Figure 2F). Another highly conserved residue in fungi MTF1 proteins, E144, forms H-bond with the NT -3 base. Even though the NT -4 base has no specific polar interaction, a purine ring at this position favors optimum base stacking interactions. 
The residues in NT groove that are involved in the specific recognition of -1 to -4 NT bases are conserved in MTF1 proteins of a wide range of fungi, including infectious Candida species (Figure S5A) but not in TFB2M. Moreover, the crystal structure of h-mtRNAP IC indicates that there are no base-specific interactions between TFB2M and non-template strand in the bubble. In contrast, TFB2M contains a characteristic loop (residue 155 to 166), which is not present in MTF1. This loop in TFB2M is located near the +1 to +5 NT bases of h-mt promoters and likely engaged in promotor opening and bubble formation. These significant differences in promoter recognition between $y$ - and h-mtRNAP ICs suggest that disrupting the -1 to -4 NT DNA binding sites in MTF1 can be a strategy for developing inhibitors to treat yeast infections. The set of interactions between MTF1 and AAGT sequence is reminiscent of the interaction of the sigma-2 region of bacterial sigma factors with the -10 element in bacterial RNAP initiation complexes (Jang and Jaehning, 1991; Lee and Borukhov, 2016; Lin et al., 2017).

The h-mtRNAP IC depends on TFAM for transcription initiation. However, y-mtRNAP does not require the TFAM homolog ABF2 (Parisi et al., 1993). Our structure provides new insights into why y-mtRNAP does not require ABF2 for transcription initiation: (i) In ymtRNAP structures, the elements such as AT-rich loop, specificity loop, and NT-groove interact with nine-nucleotide promoter sequence and (ii) the characteristic CTD insertion in $y$-mtRNAP interacts with the downstream DNA promoter to stabilize the initiation complex. The h-mtRNAP IC structure does not show analogous interactions, and TFAM apparently compensates the loss of interaction and stabilizes h-mtRNAP IC.

\section{Biochemical validation of PmIC}

We show that bubble promoter DNA used in the structural studies is catalytically active in making runoff RNA products (Figure S4I). We mutated a few promoter interacting and noninteracting residues of MTF1 to validate the MTF1:DNA interactions. The E144 lies close to the promoter DNA, and structure indicates that mutation of E144 to F, which is a bulky aromatic side chain, can interfere with the binding of NT -3 and -4 bases (Figures $2 \mathrm{~F}-\mathrm{G}$ ). The residues R178 and K179 are close to the promoter DNA, and double mutation R178A+K179A can reduce DNA-backbone interactions; K179 is strictly conserved in MTF1 proteins. The 
C192F mutation, that was reported to cause transcription defect (Karlok et al., 2002), appears to have analogous structural impact as E144F mutation (Figure 2F); as negative controls, we made MTF1 mutations, E141F and N211A + K214A, which do not interact with DNA. Additionally, in vitro transcription initiation reactions show that E144F and $\mathrm{R} 178 \mathrm{~A}+\mathrm{K} 179 \mathrm{~A}$ mutant MTF1 proteins are about 80\% less efficient in synthesizing runoff RNA products in comparison to the negative controls (Figure $2 \mathrm{H}$ ).

To determine if the defect in runoff synthesis in E144F and R178A+K179A mutants is due to DNA melting or initiation complex formation, we used previously developed 2-aminopurine based assays to interrogate these steps of transcription (Tang et al., 2009). Both MTF1 mutants showed evidence for the melting of -4 base pair; however, they showed a defect in initiation complex formation as evident from weak binding of the initiating NTPs (Figure S5B-C). The 2-AP experiments suggest that the bulky aromatic substitution of E144 and the loss of backbone interactions with K179 alters the bubble DNA conformation, which interferes with the formation of a productive IC state and reflected in lower transcription runoff RNA synthesis (Figure $2 \mathrm{H}$ ).

\section{The initiation complex shows template alignment and non-template DNA scrunching}

The $3.7 \AA$ structure of y-mtRNAP IC shows binding of a 2-mer RNA and an incoming NTP (Figure 3A). Comparison of the PmIC and IC states reveals the mechanism of template alignment and RNA synthesis during initiation. The IC structure also resolves many previously uncharacterized structural elements, including the C-tail of MTF1 and the scrunched NT DNA strand (Figures 3B-C, stereo view of the density map in Figure S6A). Comparison of the PmIC and IC structures shows that 2-mer RNA and NTP binding expands the pseudo 4-nucleotide bubble in the PmIC structure to a 7-nucleotide transcription bubble in IC. Upstream promoter involving the intercalating hairpin, specificity loop, and the MTF1 NT-groove in the PmIC structure remain intact in IC. In contrast, the position and conformation of the downstream DNA, and DNA interacting regions of $y$-mtRNAP CTD beyond the thumb subdomain undergo significant conformational changes including the closing of the fingers subdomain around the NTP-binding site (Movie S1). During the transition from PmIC to IC, the template strand undergoes a large conformational switching 
to get aligned with the 2-mer RNA and the UTP $\alpha$ S at the active site (Figure 3C; Movie S2). In the IC state, the A:T base-pair at position +3 unwinds, and the melted adenine translocates into the active site to base paired with the incoming UTP $\alpha$ S. Meanwhile, the upstream DNA remains stably bound to y-mtRNAP and MTF1. The unsynchronized events at the upstream and downstream ends of the transcription bubble result in scrunching of the NT strand to create an NT-loop preceding the -1 position (Movie S3).

DNA scrunching has been proposed as an underlying consequence of the mechanism of transcription initiation by DNA-dependent RNAPs, including y-mtRNAP (Cheetham and Steitz, 1999; Kapanidis et al., 2006; Sohn et al., 2020; Tang et al., 2008). However, the conformation of the scrunched DNA has not been captured in any of the high-resolution structures, and our y-mtRNAP IC structure is the first to capture the scrunched DNA conformation (Figure 3C). The looping of the NT strand in IC alters the track of the downstream DNA and bends the DNA more sharply from $\sim 60^{\circ}$ in PmIC to $\sim 120^{\circ}$ in IC with respect to the upstream DNA (Figures 3D-E). The scrunched NT-loop is stabilized by interacting with the structural elements of $y$-mtRNAP and MTF1, including the intercalating hairpin residues $\mathrm{H} 641$ and N642, thumb residues R780 and K787, and MTF1 C-tail residues M334 and Y335. These interactions are expected to reduce the energy of the system acquired through scrunching of the DNA in IC states, thus partially easing the instability of the intermediate IC states.

\section{The MTF1 C-tail plays a central role in transcription initiation}

Both MTF1 and TFB2M contain a structurally flexible C-terminal tail (C-tail). A recent biochemical study indicated that the C-tail plays an important role in autoinhibiting the DNA binding activity of free MTF1, and in aligning the template strand during transcription initiation (Basu et al., 2020). The biochemical studies also suggested that the MTF1 C-tail is essential for stabilizing the scrunched DNA conformation, and deletion of the C-tail decreases abortive synthesis and delays the transition into elongation. The y-mtRNAP IC structure provides the structural basis to understand the multipurpose role of the MTF1 Ctail. The structure shows that the flexible C-tail of MTF1 (D326-G341) is guided towards the active site by the MTF1/B2 hairpin (Figure S4E). In both PmIC and IC structures, the base of 
the C-tail (L328 - M334) is stabilized by the intercalating hairpin, the thumb helix, and a loop (S521 - I526) of y-mtRNAP (Figure 4A). Importantly, the tip of the C-tail (T337 - G341), which was disordered in the PmIC structure, is now ordered in the IC structure by accruing new interactions with the 5'-end of the RNA transcript, template DNA, and scrunched NTloop of the expanded transcription bubble. The C-tail tip residue S340 is at a distance of 3.8 $\AA$ A from the 5'-end $\alpha$-phosphate of pppGpG RNA. The main chain carbonyl of E338 forms Hbond with the $\mathrm{N} 1$ and $\mathrm{N} 6$ atoms of the template -2 adenine base; we expect that the N3 and $\mathrm{N} 4$ atoms of the consensus cytosine at -2 position will make similar interactions; proteinDNA crosslinking studies have shown that the $C$-tail is in the proximity of the $-3 /-4$ base of the template strand (Drakulic et al., 2014; Savkina et al., 2010). The C-tail also stabilizes the scrunched NT-loop; M334-Y335 of C-tail form almost a flat wall that stacks against the looped-out +1 and +2 bases of the NT loop (Figure 4B). The amino acid sequence conservation at the base of the C-tail is high; however, the sequence of the C-tail tip region varies across fungi species (Figure S5A).

Our structure-based projections indicate that the MTF1 C-tail will be progressively pushed away from its position in IC as the RNA:DNA hybrid grows and the NT-loop expands with RNA synthesis (Figure 4C). At a critical length of RNA, the C-tail will be displaced out of the active-site cavity. Single-molecule FRET studies of y-mtRNAP IC show that the transition from initiation to an early elongation state completes at 8-mer RNA synthesis, and MTF1 Ctail deletion delays this transition (Basu et al., 2020; Sohn et al., 2020). The superposition of $y$-mtRNAP IC and h-mtRNAP IC structures reveals that h-mtRNAP and TFB2M superimpose reasonably well on y-mtRNAP and MTF1, respectively (Figures S6B-C), and their respective promoter DNAs are aligned as well (Figure S6D). Upon transition, both elongation complexes are expected to have analogous structural states with superimposable mtRNAP, up- and down-stream DNA, RNA/DNA, and exiting RNA. In the absence of an elongation complex structure of $y$-mtRNAP, we superimposed the $y$-mtRNAP IC structure on the elongation complex structure of h-mtRNAP (Schwinghammer et al., 2013), by aligning the mtRNAP subunits. The positioning of structural elements shows that the C-tail must exit from the active site cavity prior to the complete transition into the elongation complex (Figure 4D). Upon total displacement of the C-tail, the MTF1/B2 hairpin switches its role from guiding the 
C-tail in IC to supporting the upstream DNA, as seen in the h-mtRNAP elongation complex structure.

\section{Abortive synthesis - the role of scrunching/unscrunching dynamics and steric clashes}

During transcription initiation, short RNA transcripts often dissociate as abortive products from the IC states, and all DNA-dependent RNAPs demonstrate abortive synthesis. The ymtRNAP IC produces relatively large amounts of 2-mer and 3-mer abortive products compared to other short RNAs (Figure 2H). The PmIC and IC structures provide the basis for understanding the mechanism of abortive synthesis. Two mutually non-exclusive models have been proposed for abortive synthesis: (i) Abortive RNAs are produced because of scrunching/unscrunching transitions during transcription initiation (Revyakin et al., 2006). It is argued that the scrunched state is energetically unstable, and relaxation to the more stable unscrunched state releases short RNAs as abortive products. (ii) Abortive RNAs are generated by steric clashes between the progressively growing RNA:DNA and structural elements such as the thumb subdomain of T7 RNAP (Cheetham and Steitz, 1999), the C-tail of mitochondrial transcription factors (Basu et al., 2020), or sigma-3.2/B-finger of multisubunit transcription factors (Liu et al., 2011; Murakami et al., 2002). Our structures support both mechanisms.

The co-existence of scrunched IC and unscrunched PmIC states in the cryo-EM sample suggests continuous release and reloading of 2-mer RNA without disengaging the RNAP from the promoter DNA (Movie S2). This observation is consistent with single-molecule fluorescence studies showing that T7 and bacterial RNAPs remain stably bound to the promoter during abortive synthesis (Koh et al., 2018; Revyakin et al., 2006). Continuous scrunching/unscrunching, that we infer based on the coexistence of both states in solution, is consistent with a recent single-molecule FRET study of y-mtRNAP where dynamic switching between scrunched and unscrunched states was observed throughout transcription initiation (Sohn et al., 2020). The dynamic equilibrium between IC and PmIC states in our study suggests that in an abortive event, the scrunched IC state switches to the unscrunched PmIC state, which will then rebind the NTPs to start a fresh cycle of RNA 
synthesis. The absence of natural NTPs in our sample forbids RNA synthesis, and therefore y-mtRNAP:MTF1:DNA complex ends up switching between the PmIC and IC states by constant loading and unloading of 2-mer RNA and UTP $\alpha$ S.

Our y-mtRNAP IC structure also predicts that the MTF1 C-tail is in the path of the growing RNA and will sterically clash with the RNA:DNA hybrid and the NT-loop as these elements grow in size with RNA synthesis (Figure 4C). To accommodate the longer RNA:DNA and NTloop, the C-tail must move away and subsequently exit to facilitate switching from initiation to elongation state. When the C-tail resists, steric clashes could trigger dissociation of the RNA transcript as an abortive product. The steric clash model is consistent with recent biochemical studies that showed reduced abortive synthesis when MTF1 C-tail was deleted (Basu et al., 2020).

\section{The IC structure captures the catalytic state poised for nucleotide incorporation}

Both $y$-mtRNAP and MTF1 protein residues make extensive interactions with the transcription bubble in the y-mtRNAP IC structure, shown schematically in Figure 5A. The ymtRNAP IC structure reveals the mode of binding of an NTP poised for incorporation. We used UTP $\alpha$ S to obtain (i) better metal chelation at the active site than that of a nonhydrolysable NTP analog and (ii) slower incorporation as compared to a natural NTP. The $\mathrm{UTP} \alpha \mathrm{S}$ sample was a racemic mixture, and the density favors the Sp isomer (Figure 5B). The active site regions of single-subunit RNAPs are highly conserved (Figure S3A). The ymtRNAP IC shows that the 2-mer RNA and UTP $\alpha$ S, which are base-paired with the template +1 to +3 nucleotides, are secured by extensive interactions with conserved palm subdomain residues. These interactions are maintained in the elongation phase (Yin and Steitz, 2004). One oxygen from each of the three phosphates of UTP $\alpha \mathrm{S}$ chelates a $\mathrm{Mg}^{2+}$ ion in the active site (Figure 5C). The metal chelation of $\mathrm{UTP} \alpha \mathrm{S}$ is reminiscent of the coordination of metal-ion $\mathrm{B}$ with NTP/dNTP (Steitz, 1999).

The O-helix (residues 1010 - 1025) in the fingers subdomain plays an essential role in binding NTPs (Figure S7A). The residue Y1022, the positional and functional equivalent of T7 RNAP residue Y639, interacts with the 2'-OH of the incoming UTP $\alpha$ S and provides specificity for binding rNTPs over dNTPs (Sousa and Padilla, 1995; Yin and Steitz, 2004). The conformation of 
Y1022 in y-mtRNAP is distinct from that of Y639 in T7 RNAP IC, which represents a posttranslocated state with no bound NTP (Figure 5C). This conserved tyrosine toggles between the two positions during each cycle of the NTP binding, nucleotide incorporation, and translocation. The template DNA is bent sharply at positions -1 and +3 in the active site of $y$-mtRNAP IC (Figure 5D; stereo view, Figure S7B). A similarly bent template DNA conformation is present in T7 RNAP IC structure (Cheetham and Steitz, 1999; Kennedy et al., 2007), which suggests that this feature of the template DNA is conserved in single-subunit RNAP ICs. The Y-helix (1023-1041) is critical for unwinding the downstream DNA (Figure S7A). Based on active-site superposition of h- and $y$-mtRNAP IC structures, the Y-helix of h-mtRNAP clashes with the template strand of y-mtRNAP IC active complex, and the clash explains why the h-mtRNAP IC structure that represents an inactive-clenched state is not compatible with NTP-binding (Figure 5E). The binding of nonhydrolysable AMPCPP to T7 RNA polymerase active site (Yin and Steitz, 2004) and UTP $\alpha$ S to y-mtRNAP in the IC structure have few key differences (Figure S7C).

\section{Mitochondrial toxicity by antiviral nucleosides/nucleotides}

Nucleos(t)ide analogs are widely used to treat viral infections but they cause cytotoxicity by inhibiting transcription by cellular RNAPs and h-mtRNAP. The NTP-binding sites of h- and ymtRNAP are fully conserved, and in the absence of a catalytic complex structure of h-mtRNAP, y-mtRNAP IC structure can be used to model the binding of NTP analogs (Figures 6A-B). Remdesivir is a nucleotide analog with broad antiviral profile (Warren et al., 2016) including the treatment of SARS-CoV-2 (COVID-19) infection. Modeling of remdesivir-diphosphate, the active metabolite, into the NTP-binding pocket of mtRNAP reveals that the characteristic 1'-cyano group of remdesivir clashes with the conserved H1125 in h-mtRNAP (Figure 6B). Thus, remdesivir is expected to have low cytotoxicity, which is consistent with its low incorporation efficiency by hmtRNAP (Tchesnokov et al., 2019; Warren et al., 2016). Recent structures of SARS-CoV-2 RdRp bound to RNA substrates (Hillen et al., 2020; Yin et al., 2020) have paved the path for designing target-specific nucleos(t)ide analogs, and evaluation of mitochondrial toxicity of inhibiters can play a valuable role. 


\section{Limitations}

The N-terminal extension domain (NTE) is disordered in the y-mtRNAP structures. The density for the characteristic C-terminal insert in y-mtRNAP is lower than 4 Å resolution, and therefore the insert has missing stretches and side chains. The NTE may contain additional promoter binding elements, which along with the unique insert in y-mtRNAP may play crucial roles in stabilizing the initiation complexes. The pre-melted promoter used in our studies did not reveal the mechanism of early steps of transcription initiation, including closed complex formation and transition into PmIC.

\section{ACKNOWLEDGMENTS}

We thank Electron Cryogenic Microscopy, Brussels, Thermo Fisher Scientific, Eindhoven, and ESRF-Grenoble for microscope access, and Marcus Fislage, Adrian Koh and Abhimanyu Singh for helpful discussion. This research was supported by NIH grant R35 GM118086 to SSP, and KU Leuven start-up and Rega Virology and Chemotherapy internal grants to KD.

\section{AUTHOR CONTRIBUTIONS}

B.D.W.; S.S.; A.S., H.V.; J.S.; D.V.; S.E.M.; KD investigation and validation; B.D.W; S.S.; S.S.P.; K.D. conceptualization; C.D.; E.K. resources; B.D.W.; E.K.; S.S.P.; K.D. data curation; S.S.P.; K.D. supervision, writing, editing, visualization, funding acquisition, and project administration.

\section{DECLARATION OF INTERESTS}

Authors declare no competing interests. 


\section{TABLE LEGEND}

Table 1. Single particle cryo-EM data and structure analysis statistics.

\section{FIGURE LEGENDS}

\section{Figure 1. Structure of yeast mitochondrial RNA polymerase (y-mtRNAP).}

(A) A schematic 1D representation of $y$-mtRNAP and comparison of its key structural elements with those of h-mtRNAP and T7 RNAP.

Side-by-side comparison of (B) y-mtRNAP, (C) h-mtRNAP (PDB Id. 6EQR) (D) T7 RNAP (PDB Id. 1QLN) structures in their respective transcription initiation states. The template (T) and non-template (NT) DNA strands are shown in pink and cyan surface representations, respectively. The C-terminal domains (CTDs) of all single-stranded RNA polymerases are shaped like a right-hand with functionally conserved structural elements, including the thumb, fingers, and palm subdomain shown in green, blue, and red ribbons, respectively. The key structural elements involved in promoter recognition and binding, such as AT-rich recognition loop (black), intercalating hairpin (ICH, dark blue), specificity loop (SL, magenta) and MTF1/B2 hairpin (orange) are indicated. The transcription factors MTF1 and TFB2M are in yellow, and TFAM and penta-tricopeptide repeat (PPR) domain in h-mtRNAP IC are in light brown and light green, respectively. The RNA and NTP are represented as green and magenta sphere models, respectively.

Figure 2. Structure of the yeast mitochondrial RNA polymerase partially-melted transcription initiation complex (y-mtRNAP PmIC).

(A) Nucleotide sequence of the pre-melted initiation bubble with $15 S$ yeast mtDNA promoter sequence. The bubble was created by mutating the -4 to +2 nucleotides of the template strand; missing nucleotides in the structure are in gray.

(B) Unsharpened $3.1 \AA$ Å resolution density map shows the DNA track in the y-mtRNAP PmIC structure; blue y-mtRNAP, yellow MTF1, cyan non-template (NT), and pink template (T).

(C) B-factor putty (thin blue, lowest to thick red, highest B-factors ranging from 30 to 200 $\AA^{2}$ ) representation of DNA in the active-site cavity; MTF1 (yellow) and y-mtRNAP (gray), 
intercalating hairpin (ICH), specificity loop (SL), non-template (NT) groove and MTF1 C-tail are highlighted.

(D) The interactions of -4 to -1 NT nucleotides with the NT-groove in MTF1 (yellow surface). The density map defines the track of the NT nucleotides; the boxed regions are elaborated in panels E and F.

(E) Aromatic ring stacking and base-specific H-bond interactions of the -2 NT guanine base with the NT groove residues (stereo view, Figure S4H); N1 and N2 interact with the mainchain carbonyl of Y103, 06 with the mainchain amide of W105, and N1 and N3 interact with $0 \varepsilon 1$ of Q149 sidechain (Figure S4H).

(F) Position and interactions of -3 and -4 NT bases; the mutation C192F appears to impact transcription initiation (Karlok et al., 2002) by interfering with -3 and -4 NT nucleotides.

(G) MTF1 residues in and around the NT groove that were mutated to ascertain their role in transcription activity.

(H) Yeast mitochondrial 21S rRNA promoter fragment with highlighted start-site (top). Image of the polyacrylamide gel shows abortive and runoff RNA products after 15 min of transcription reaction with y-mtRNAP, MTF1 WT or mutants. Lane 2 shows the 2-mer RNA marker from a reaction performed with ATP and GTP spiked with $\alpha-32$ P-GTP. The reactions in the rest of the lanes contained ATP, UTP, GTP, and 3'-dCTP. (Below, right) The bar chart shows the \% of runoff RNA synthesized by MTF1 WT and mutants. Error bars represent standard deviations from two experiments (Below, right).

\section{Figure 3. Structure of the yeast mitochondrial RNA polymerase transcription initiation complex (y-mtRNAP IC).}

(A) The pre-melted initiation bubble DNA template (T, pink) and non-template (NT, cyan), pppGpG RNA (green), and a non-hydrolysable UTP $\alpha$ S (magenta) used in assembling ymtRNAP IC; binding of UTP $\alpha$ S unwinds the downstream +3 base-pair and engages +3 template nucleotide base-paired to form a catalytic-competent quinary IC.

(B) The $3.7 \AA$ resolution density map defines the position and conformation of the transcription bubble, 2-mer RNA, and UTP $\alpha$ S; stereo view, Figure S6A. 
(C) Superposition of PmIC and IC structures shows the large conformational changes (indicated by arrows) in the transcription bubble from black NT and gray T strands in PmIC to cyan NT and pink T strands in IC, respectively (Movie S2); the region highlighted by dotted red circle shows the scrunching of NT strand as a loop (Movie S3).

(D) Comparison of experimental cryo-EM density maps of PmIC (gray) and IC (orange) shows that the downstream DNA bends further and fingers close-in as PmIC switches to IC (Movie S1); the blue arrows indicate the motion of downstream DNA and fingers.

(E) Alignment of the upstream promoter DNA in PmIC and IC shows the increased bend in the downstream DNA of the IC ( $\sim 180^{\circ}$ switched view of panel C). The angle between the upstream and downstream DNA are about $120^{\circ}$ and $60^{\circ}$, respectively, in PmIC (blue axes) and IC (red axes); i.e., the DNA is bent by $\sim 60^{\circ}$ in PmIC and subsequently by additional $60^{\circ}$ to $\sim 120^{\circ}$ in the IC structure. The DNA bending calculations were done using CURVES+ server.

Figure 4. Multipurpose role of the MTF1 C-tail in stabilizing the y-mtRNAP IC, triggering abortive synthesis, and triggering transition from IC to an elongation complex.

(A) The MTF1 C-tail defined by $3.7 \AA ̊$ cryo-EM map takes a central position in y-mtRNAP IC (Figure S3A). The C-tail interacts with the intercalating hairpin (ICH), thumb helix, template (T), non-template (NT), and the RNA transcript, pppGpG. The N1 and N6 atoms of the -2 template nucleotide form H-bonds with the mainchain carbonyl of E338, as indicated by dotted lines. The sidechain of S340 is in close contact (3.8 $\AA$ ) with $\alpha$-phosphate of pppGpG RNA.

(B) A zoomed view of the M334-Y335 platform in MTF1 C-tail stacking against the +1 and +2 NT nucleotides of the NT-loop.

(C) The NT strand in y-mtRNAP PmIC (black) grows towards the C-tail and adapts a loop conformation (cyan) in y-mtRNAP IC. As RNA synthesis progresses, expansion of scrunched NT-loop and the growing RNA:DNA hybrid will push (red arrows) the MTF1 C-tail and trigger a large conformational change that is required for transition from IC to elongation complex (EC) . 
(D) Superposition of y-mtRNAP IC on h-mtRNAP EC (PDB Id. 4BOC) shows that the transition from IC (pink template and green RNA) to EC (black template and gray RNA) requires ejection of the MTF1 C-tail. The arrows indicate repositioning of the template in the transition from IC to EC.

Figure 5. Network of transcription bubble interactions in y-mtRNAP IC structure and comparison of the active site region of $y$-mtRNAP IC with T7 RNAP IC and h-mtRNAP IC.

(A) A schematic representation of the interactions of the transcription bubble with surrounding protein residues of MTF1 (black) and y-mtRNAP (blue); the non-template strand, template strand, pppGpG (RNA) and UTP $\alpha$ S are in cyan, pink, green, and magenta, respectively.

(B) The chemical structure of UTP $\alpha$ S Sp isomer that binds to y-mtRNAP IC.

(C) A superposition of NTP bound y-mtRNAP IC (pink) on T7 RNAP IC (PDB ID. 1QLN) (gray) structure. The y-mtRNAP IC has captured the incoming UTP $\alpha$ S in a catalytic mode for nucleotide incorporation whereas the T7 RNAP IC structure represents the posttranslocated state with no bound NTP. The superposition shows that the NTP-binding pocket undergoes a conformational change to bind NTP following translocation; the conserved Y639 on the O helix of T7 RNAP must shift to the position that Y1022 of y-mtRNAP takes to accommodate an NTP. The repositioning of Y639 (as indicated by arrows) accompanies the shifting of the template base into the active site to base-pair with NTP.

(D) The superposition shows that the promoter DNA templates in y-mtRNAP IC (pink) and T7 RNAP (gray) are bent sharply at the active site in a similar manner between positions -1 and +3 (Stereo view Figure S7B).

(E) Superposition of y-mtRNAP IC on h-mtRNAP IC structure (PDB Id. 6EQR) shows that the Y-helix of h-mtRNAP clashes with the template strand of y-mtRNAP IC, and the state observed in the h-mtRNAP IC can't position the template in a conformation that is compatible for RNA/NTP-binding. Thus, the h-mtRNAP IC structure represents an inactive-clenched state. The Y- helix (1023-1041 of y-mtRNAP) is critical for downstream DNA unwinding (Figure S7A). 
Figure 6. Bound UTP $\alpha$ S and modeled remdesivir-diphosphate (DP) in the NTP-binding pocket of IC. (A) The $3.7 \AA$ density map of y-mtRNAP IC defines the binding of UTP $\alpha$ S in a catalytic competent mode. Key y-mtRNAP residues (h-mtRNAP equivalents are in parenthesis) that interact with NTP and RNA:DNA hybrid are listed; R827 (R803), R829 (R805), Y831 (Y807), Y979 (Y956), K1014 (K991), M1018 (M995), and Y1022(Y999).

(B) Modeling shows incompatibility of remdesivir-DP (cyan) binding in the pocket primarily due to a steric clash of the 1'-cyano moiety of the inhibitor with the conserved H1125 of hmtRNAP. 


\section{STAR*METHODS}

Detailed methods are provided in the online version of this paper and include the following:

- KEY RESOURCES TABLE

- CONTACT FOR REAGENT AND RESOURCE SHARING

- METHOD DETAILS

- Expression and purification of MTF1

- Expression and purification of yeast mitochondrial RNA polymerase (y-mt RNAP)

- In vitro transcription initiation assay to measure runoff and abortive RNA synthesis

○ 2-aminopurine assays for DNA melting and initiating NTP binding

○ Ultrafiltration assay to monitor y-mtRNAP - MTF1 interaction

- Assembly partially-melted initiation(PmIC) complex and initiation complex (IC).

- Multi-angle and dynamic light scattering studies of PmIC and IC.

o Thermal shift assays showing the stability of PmIC and IC.

○ Single particle cryo-EM structure determination of y-mtRNAP PmIC

- Single particle cryo-EM structure determination of y-mtRNAP IC.

- DATA AND SOFTWARE AVAILABILITY

"Mitochondria RNA polymerase runoff RNA gel", Mendeley Data, V2, doi: 10.17632/vtd45jkx6v.2 


\section{SUPPLEMENTAL MOVIES}

Movie S1. Overall structural change in the transition from PmIC to IC (related to Figure 3). Morphing between the transcription partially-melted initiation state (PmIC, gray y-mtRNAP and DNA, yellow MTF1) and initiation state (IC, yellow MTF1, blue y-mtRNAP, cyan non-template, and pink template) simulates the conformational changes in the promoter and proteins during transition from the PmIC to IC state. The downstream DNA bends inward and parts of the Cterminal domain of y-mtRNAP including fingers (in front) undergo large conformational changes. MTF1, upstream DNA, and the N-terminal domain of y-mtRNAP that interact with MTF1 and upstream DNA show minimal conformational changes; e.g. the thumb helix on the left and MTF1 on the top have minimal movements.

\section{Movie S2. Conformational change of downstream DNA and expansion of the transcription} bubble (related to Figure 3). Morphing between the PmIC state (gray DNA) and IC state (cyan non-template, pink template, and 2-mer RNA pppGpG and UTP $\alpha$ S in stick models) shows that the DNA bubble expanded, and the template is base-paired with the 2-mer RNA and UTP at the polymerase active site. The downstream DNA bends further by about $60^{\circ}$ with respect to the upstream DNA (Figure 3E). Protein atoms are omitted for clear visualization of the DNA.

Movie S3. Scrunching of the non-template DNA strand as an NT-loop (related to Figure 3). Morphing between the PmIC state (gray DNA) and IC state (cyan non-template, pink template, and 2-mer RNA pppGpG and UTP $\alpha$ S in stick models) shows looping of the non-template strand into an NT-loop. This looping appears to be a major contributor to bending of the downstream DNA with respect to the upstream DNA (Figure 3E). 


\section{SUPPLEMENTAL INFORMATION}

Supplemental Information contains seven figures (S1 -S7).

- Figure S1: Biophysical characterization of y-mtPmIC; related to Table 1 and Figure 2.

- Figure S2. Cryo-EM data processing and density maps; related to Table 1.

- Figure S3. Amino acid sequence comparison of single-subunit RNA polymerases; related to Figure 1.

- Figure S4. Structural and biochemical analysis of interactions among y-mtRNAP, MTF1, and DNA; related to Figure 2.

- Figure S5. Amino acid sequence comparison of fungal MTF1 proteins and 2-AP promoter melting and initiating NTP binding studies of mutant MTF1; related to Figures 2 and 4.

- Figure S6. Comparison of y-mtRNAP IC and h-mtRNA IC structures; related to Figure 5.

- Figure S7. Positioning of the template, RNA and NTP at the active site region in ymtRNAP IC structure; related to Figures 5 and 6. 


\section{REFERENCES}

Basu, U., Lee, S.W., Deshpande, A., Shen, J., Sohn, B.K., Cho, H., Kim, H., and Patel, S.S. (2020). The C-terminal tail of the yeast mitochondrial transcription factor Mtf1 coordinates template strand alignment, DNA scrunching and timely transition into elongation. Nucleic Acids Res 48, 2604-2620.

Biswas, T.K., and Getz, G.S. (1986). A critical base in the yeast mitochondrial nonanucleotide promoter. Abolition of promoter activity by mutation at the -2 position. J Biol Chem 261, 3927-3930.

Biswas, T.K., Ticho, B., and Getz, G.S. (1987). In vitro characterization of the yeast mitochondrial promoter using single-base substitution mutants. J Biol Chem 262, 1369013696.

Blanchet, C., Pasi, M., Zakrzewska, K., and Lavery, R. (2011). CURVES+ web server for analyzing and visualizing the helical, backbone and groove parameters of nucleic acid structures. Nucleic Acids Res 39, W68-73.

Browning, D.F., and Busby, S.J. (2004). The regulation of bacterial transcription initiation. Nat Rev Microbiol 2, 57-65.

Cheetham, G.M., and Steitz, T.A. (1999). Structure of a transcribing T7 RNA polymerase initiation complex. Science 286, 2305-2309.

Cheetham, G.M., and Steitz, T.A. (2000). Insights into transcription: structure and function of single-subunit DNA-dependent RNA polymerases. Curr Opin Struct Biol 10, 117-123. Chen, J., Chiu, C., Gopalkrishnan, S., Chen, A.Y., Olinares, P.D.B., Saecker, R.M., Winkelman, J.T., Maloney, M.F., Chait, B.T., Ross, W., et al. (2020). Stepwise Promoter Melting by Bacterial RNA Polymerase. Mol Cell 78, 275-288 e276.

Cramer, P. (2019). Organization and regulation of gene transcription. Nature 573, 45-54. de la Rosa-Trevin, J.M., Quintana, A., Del Cano, L., Zaldivar, A., Foche, I., Gutierrez, J., GomezBlanco, J., Burguet-Castell, J., Cuenca-Alba, J., Abrishami, V., et al. (2016). Scipion: A software framework toward integration, reproducibility and validation in 3D electron microscopy. J Struct Biol 195, 93-99.

Deshpande, A.P., and Patel, S.S. (2012). Mechanism of transcription initiation by the yeast mitochondrial RNA polymerase. Biochim Biophys Acta 1819, 930-938.

Drakulic, S., Wang, L., Cuellar, J., Guo, Q., Velazquez, G., Martin-Benito, J., Sousa, R., and Valpuesta, J.M. (2014). Yeast mitochondrial RNAP conformational changes are regulated by interactions with the mitochondrial transcription factor. Nucleic Acids Res 42, 1124611260.

Emsley, P., and Cowtan, K. (2004). Coot: model-building tools for molecular graphics. Acta Crystallogr D Biol Crystallogr 60, 2126-2132.

Falkenberg, M., Gaspari, M., Rantanen, A., Trifunovic, A., Larsson, N.G., and Gustafsson, C.M. (2002). Mitochondrial transcription factors B1 and B2 activate transcription of human mtDNA. Nat Genet 31, 289-294.

Fisher, R.P., Lisowsky, T., Parisi, M.A., and Clayton, D.A. (1992). DNA wrapping and bending by a mitochondrial high mobility group-like transcriptional activator protein. J Biol Chem 267, 3358-3367.

Gustafsson, C.M., Falkenberg, M., and Larsson, N.G. (2016). Maintenance and Expression of Mammalian Mitochondrial DNA. Annu Rev Biochem 85, 133-160. 
Hillen, H.S., Kokic, G., Farnung, L., Dienemann, C., Tegunov, D., and Cramer, P. (2020). Structure of replicating SARS-CoV-2 polymerase. Nature 584, 154-156.

Hillen, H.S., Morozov, Y.I., Sarfallah, A., Temiakov, D., and Cramer, P. (2017). Structural Basis of Mitochondrial Transcription Initiation. Cell 171, 1072-1081 e1010.

Hillen, H.S., Temiakov, D., and Cramer, P. (2018). Structural basis of mitochondrial transcription. Nat Struct Mol Biol 25, 754-765.

Huynh, K., and Partch, C.L. (2015). Analysis of protein stability and ligand interactions by thermal shift assay. Curr Protoc Protein Sci 79, 2829 21-28 2914.

Imburgio, D., Rong, M., Ma, K., and McAllister, W.T. (2000). Studies of promoter recognition and start site selection by T7 RNA polymerase using a comprehensive collection of promoter variants. Biochemistry 39, 10419-10430.

Jang, S.H., and Jaehning, J.A. (1991). The yeast mitochondrial RNA polymerase specificity factor, MTF1, is similar to bacterial sigma factors. J Biol Chem 266, 22671-22677.

Kandiah, E., Giraud, T., de Maria Antolinos, A., Dobias, F., Effantin, G., Flot, D., Hons, M., Schoehn, G., Susini, J., Svensson, O., et al. (2019). CM01: a facility for cryo-electron microscopy at the European Synchrotron. Acta Crystallogr D Struct Biol 75, 528-535. Kapanidis, A.N., Margeat, E., Ho, S.O., Kortkhonjia, E., Weiss, S., and Ebright, R.H. (2006). Initial transcription by RNA polymerase proceeds through a DNA-scrunching mechanism. Science 314, 1144-1147.

Karlok, M.A., Jang, S.H., and Jaehning, J.A. (2002). Mutations in the yeast mitochondrial RNA polymerase specificity factor, Mtf1, verify an essential role in promoter utilization. J Biol Chem 277, 28143-28149.

Kennedy, W.P., Momand, J.R., and Yin, Y.W. (2007). Mechanism for de novo RNA synthesis and initiating nucleotide specificity by t7 RNA polymerase. J Mol Biol 370, 256-268.

Kim, H., Tang, G.Q., Patel, S.S., and Ha, T. (2012). Opening-closing dynamics of the mitochondrial transcription pre-initiation complex. Nucleic Acids Res 40, 371-380.

Koh, H.R., Roy, R., Sorokina, M., Tang, G.Q., Nandakumar, D., Patel, S.S., and Ha, T. (2018). Correlating Transcription Initiation and Conformational Changes by a Single-Subunit RNA Polymerase with Near Base-Pair Resolution. Mol Cell 70, 695-706 e695.

Kucukelbir, A., Sigworth, F.J., and Tagare, H.D. (2014). Quantifying the local resolution of cryo-EM density maps. Nat Methods 11, 63-65.

Lee, J., and Borukhov, S. (2016). Bacterial RNA Polymerase-DNA Interaction-The Driving

Force of Gene Expression and the Target for Drug Action. Front Mol Biosci 3, 73.

Liebschner, D., Afonine, P.V., Baker, M.L., Bunkoczi, G., Chen, V.B., Croll, T.I., Hintze, B., Hung, L.W., Jain, S., McCoy, A.J., et al. (2019). Macromolecular structure determination using Xrays, neutrons and electrons: recent developments in Phenix. Acta Crystallogr D Struct Biol 75, 861-877.

Lin, W., Mandal, S., Degen, D., Liu, Y., Ebright, Y.W., Li, S., Feng, Y., Zhang, Y., Mandal, S., Jiang, Y., et al. (2017). Structural Basis of Mycobacterium tuberculosis Transcription and Transcription Inhibition. Mol Cell 66, 169-179 e168.

Lipinski, K.A., Puchta, O., Surendranath, V., Kudla, M., and Golik, P. (2011). Revisiting the yeast PPR proteins--application of an Iterative Hidden Markov Model algorithm reveals new members of the rapidly evolving family. Mol Biol Evol 28, 2935-2948.

Liu, X., Bushnell, D.A., Silva, D.A., Huang, X., and Kornberg, R.D. (2011). Initiation complex structure and promoter proofreading. Science 333, 633-637. 
Matsunaga, M., and Jaehning, J.A. (2004). Intrinsic promoter recognition by a "core" RNA polymerase. J Biol Chem 279, 44239-44242.

McCulloch, V., Seidel-Rogol, B.L., and Shadel, G.S. (2002). A human mitochondrial transcription factor is related to RNA adenine methyltransferases and binds Sadenosylmethionine. Mol Cell Biol 22, 1116-1125.

Minton, A.P. (2016). Recent applications of light scattering measurement in the biological and biopharmaceutical sciences. Anal Biochem 501, 4-22.

Morozov, Y.I., Parshin, A.V., Agaronyan, K., Cheung, A.C., Anikin, M., Cramer, P., and Temiakov, D. (2015). A model for transcription initiation in human mitochondria. Nucleic Acids Res 43, 3726-3735.

Murakami, K.S., Masuda, S., and Darst, S.A. (2002). Structural basis of transcription initiation: RNA polymerase holoenzyme at 4 A resolution. Science 296, 1280-1284.

Nayak, D., Guo, Q., and Sousa, R. (2009). A promoter recognition mechanism common to yeast mitochondrial and phage $t 7$ RNA polymerases. J Biol Chem 284, 13641-13647. Osinga, K.A., De Haan, M., Christianson, T., and Tabak, H.F. (1982). A nonanucleotide sequence involved in promotion of ribosomal RNA synthesis and RNA priming of DNA replication in yeast mitochondria. Nucleic Acids Res 10, 7993-8006.

Paratkar, S., Deshpande, A.P., Tang, G.Q., and Patel, S.S. (2011). The N-terminal domain of the yeast mitochondrial RNA polymerase regulates multiple steps of transcription. J Biol Chem 286, 16109-16120.

Paratkar, S., and Patel, S.S. (2010). Mitochondrial transcription factor Mtf1 traps the unwound non-template strand to facilitate open complex formation. J Biol Chem 285, 39493956.

Parisi, M.A., Xu, B., and Clayton, D.A. (1993). A human mitochondrial transcriptional activator can functionally replace a yeast mitochondrial HMG-box protein both in vivo and in vitro. Mol Cell Biol 13, 1951-1961.

Pettersen, E.F., Goddard, T.D., Huang, C.C., Couch, G.S., Greenblatt, D.M., Meng, E.C., and Ferrin, T.E. (2004). UCSF Chimera--a visualization system for exploratory research and analysis. J Comput Chem 25, 1605-1612.

Posse, V., and Gustafsson, C.M. (2017). Human Mitochondrial Transcription Factor B2 Is Required for Promoter Melting during Initiation of Transcription. J Biol Chem 292, 26372645.

Ramachandran, A., Basu, U., Sultana, S., Nandakumar, D., and Patel, S.S. (2017). Human mitochondrial transcription factors TFAM and TFB2M work synergistically in promoter melting during transcription initiation. Nucleic Acids Res 45, 861-874.

Revyakin, A., Liu, C., Ebright, R.H., and Strick, T.R. (2006). Abortive initiation and productive initiation by RNA polymerase involve DNA scrunching. Science 314, 1139-1143.

Rohou, A., and Grigorieff, N. (2015). CTFFIND4: Fast and accurate defocus estimation from electron micrographs. J Struct Biol 192, 216-221.

Savkina, M., Temiakov, D., McAllister, W.T., and Anikin, M. (2010). Multiple functions of yeast mitochondrial transcription factor Mtf1p during initiation. J Biol Chem 285, 39573964.

Schwinghammer, K., Cheung, A.C., Morozov, Y.I., Agaronyan, K., Temiakov, D., and Cramer, P. (2013). Structure of human mitochondrial RNA polymerase elongation complex. Nat Struct Mol Biol 20, 1298-1303. 
Sohn, B.K., Basu, U., Lee, S.W., Cho, H., Shen, J., Deshpande, A., Johnson, L.C., Das, K., Patel, S.S., and Kim, H. (2020). The dynamic landscape of transcription initiation in yeast mitochondria. Nat Commun 11, 4281.

Sousa, R., and Padilla, R. (1995). A mutant T7 RNA polymerase as a DNA polymerase. EMBO J 14, 4609-4621.

Steitz, T.A. (1999). DNA polymerases: structural diversity and common mechanisms. J Biol Chem 274, 17395-17398.

Tang, G.Q., Deshpande, A.P., and Patel, S.S. (2011). Transcription factor-dependent DNA bending governs promoter recognition by the mitochondrial RNA polymerase. J Biol Chem 286, 38805-38813.

Tang, G.Q., Paratkar, S., and Patel, S.S. (2009). Fluorescence mapping of the open complex of yeast mitochondrial RNA polymerase. J Biol Chem 284, 5514-5522.

Tang, G.Q., Roy, R., Ha, T., and Patel, S.S. (2008). Transcription initiation in a single-subunit RNA polymerase proceeds through DNA scrunching and rotation of the $\mathrm{N}$-terminal subdomains. Mol Cell 30, 567-577.

Tchesnokov, E.P., Feng, J.Y., Porter, D.P., and Gotte, M. (2019). Mechanism of Inhibition of Ebola Virus RNA-Dependent RNA Polymerase by Remdesivir. Viruses 11.

Velazquez, G., Sousa, R., and Brieba, L.G. (2015). The thumb subdomain of yeast mitochondrial RNA polymerase is involved in processivity, transcript fidelity and mitochondrial transcription factor binding. RNA Biol 12, 514-524.

Warren, T.K., Jordan, R., Lo, M.K., Ray, A.S., Mackman, R.L., Soloveva, V., Siegel, D., Perron, M., Bannister, R., Hui, H.C., et al. (2016). Therapeutic efficacy of the small molecule GS-5734 against Ebola virus in rhesus monkeys. Nature 531, 381-385.

Yin, W., Mao, C., Luan, X., Shen, D.D., Shen, Q., Su, H., Wang, X., Zhou, F., Zhao, W., Gao, M., et al. (2020). Structural basis for inhibition of the RNA-dependent RNA polymerase from SARS-CoV-2 by remdesivir. Science 368, 1499-1504.

Yin, Y.W., and Steitz, T.A. (2004). The structural mechanism of translocation and helicase activity in T7 RNA polymerase. Cell 116, 393-404.

Zheng, S.Q., Palovcak, E., Armache, J.P., Verba, K.A., Cheng, Y., and Agard, D.A. (2017).

MotionCor2: anisotropic correction of beam-induced motion for improved cryo-electron microscopy. Nat Methods 14, 331-332.

Zivanov, J., Nakane, T., Forsberg, B.O., Kimanius, D., Hagen, W.J., Lindahl, E., and Scheres, S.H. (2018). New tools for automated high-resolution cryo-EM structure determination in RELION-3. Elife 7. 


\section{STAR*METHODS}

\section{KEY RESOURCES TABLE}

\begin{tabular}{|c|c|c|}
\hline REAGENT or RESOURCE & SOURCE & IDENTIFIER \\
\hline \multicolumn{3}{|l|}{ Antibodies } \\
\hline \multicolumn{3}{|l|}{$\mathrm{N} / \mathrm{A}$} \\
\hline \multicolumn{3}{|l|}{ Bacterial and Virus Strains } \\
\hline BL21(DE3) & Sigma-Aldrich & CMC0014 \\
\hline BL21(DE3) Codon Plus (RIL) & Agilent & $\# 230245$ \\
\hline \multicolumn{3}{|l|}{ Biological Samples } \\
\hline \multicolumn{3}{|l|}{$\mathrm{N} / \mathrm{A}$} \\
\hline \multicolumn{3}{|l|}{ Chemicals, Peptides, and Recombinant Proteins } \\
\hline Protein Thermal Shift dye for protein thermal shift assay & Applied Biosystems & Cat. \#4461146 \\
\hline Uridine $5^{\prime}(\alpha$-thio) triphosphate & Jenna Bioscience & NU-411S \\
\hline Acrylamide:Bis-Acrylamide Solution & Bio-Rad Laboratories & Cat. \# 161-0154 \\
\hline Urea & Sigma-Aldrich & Cat. \# U5378 \\
\hline Ammonium persulfate & Sigma-Aldrich & Cat. \# A3678 \\
\hline TEMED & Sigma-Aldrich & Cat. \# T9281 \\
\hline ATP, GTP and UTP & Thermo Scientific & Cat. \# R0481 \\
\hline 3'-dCTP & Trilink Biotechnologies & Cat. \# N-3003 \\
\hline$\alpha-{ }^{32} P-G T P$ & PerkinElmer & $\begin{array}{l}\text { Cat. \# } \\
\text { BLU506H250UC }\end{array}$ \\
\hline \multicolumn{3}{|l|}{ Critical Commercial Assays } \\
\hline \multicolumn{3}{|l|}{$\mathrm{N} / \mathrm{A}$} \\
\hline \multicolumn{3}{|l|}{ Deposited Data } \\
\hline $\begin{array}{l}\text { Yeast mitochondrial y-mtRNAP/MTF1/dsDNA, pre- } \\
\text { initiation complex }\end{array}$ & This paper & PDB 6YMV \\
\hline Cryo EM map for above & This paper & EMD-10845 \\
\hline $\begin{array}{l}\text { Yeast mitochondrial y-mtRNAP/MTF1/dsDNA/RNA/ } \\
\text { UTP } \alpha \text { S, initiation complex }\end{array}$ & This paper & PDB 6YMW \\
\hline Cryo EM map for above & This paper & EMD-10846 \\
\hline \multicolumn{3}{|l|}{ Experimental Models: Cell Lines } \\
\hline \multicolumn{3}{|l|}{$\mathrm{N} / \mathrm{A}$} \\
\hline Mitochondrial RNA polymerase runoff RNA gel & $\begin{array}{l}\text { doi: } \\
10.17632 / \mathrm{vtd} 45 \mathrm{jkx} 6 \\
\text { v.2 }\end{array}$ & \\
\hline \multicolumn{3}{|l|}{ Experimental Models: Organisms/Strains } \\
\hline $\mathrm{N} / \mathrm{A}$ & & \\
\hline Oligonucleotides & & \\
\hline
\end{tabular}




\begin{tabular}{|c|c|c|}
\hline $\begin{array}{l}\text { Modified yeast mitochondrial 15S promoter non- } \\
\text { template strand } \\
\text { 5' -CGAATAAGTATTGATATAAGTAATAGATAATGC }\end{array}$ & IDT & $\mathrm{N} / \mathrm{A}$ \\
\hline $\begin{array}{l}\text { Modified yeast mitochondrial } 15 S \text { promoter } \\
\text { template } \\
\text { 5' -GCATTATCTACCGACAATATCAATACTTATTCG }\end{array}$ & IDT & $\mathrm{N} / \mathrm{A}$ \\
\hline $\begin{array}{l}\text { Yeast mitochondrial } 21 S \text { rRNA promoter template } \\
\text { GGTATTTCAAATCTATTATTCTACTTTTTACTACTTAT } \\
\text { ATATATAATAATAATAATA }\end{array}$ & IDT & $\mathrm{N} / \mathrm{A}$ \\
\hline $\begin{array}{l}\text { Yeast mitochondrial } 21 S \text { rRNA promoter Non-template } \\
\text { TATTATTATTATTATATATATAAGTAGTAAAAAGTAGA } \\
\text { ATAATAGATTTGAAATACC }\end{array}$ & IDT & $\mathrm{N} / \mathrm{A}$ \\
\hline RNA sequence (primer strand): $5{ }^{\prime}$ pppGpG $3^{\prime}$ & Trilink Biotechnologies & Cat. \# O-31011 \\
\hline \multicolumn{3}{|l|}{ Recombinant DNA } \\
\hline Plasmid pTrcHisC for expressing yeast MTF1 & (Tang et al., 2009) & $\mathrm{N} / \mathrm{A}$ \\
\hline $\begin{array}{l}\text { Plasmid pProExRpo41 } \Delta \mathrm{N} 100 \text { for expressing yeast } \\
\text { RPO41 } \Delta \mathrm{N} 100\end{array}$ & $\begin{array}{l}\text { (Paratkar et al., } \\
\text { 2011) }\end{array}$ & N/A \\
\hline \multicolumn{3}{|l|}{ Software and Algorithms } \\
\hline PHENIX 1.16 & $\begin{array}{l}\text { (Liebschner et al., } \\
\text { 2019) }\end{array}$ & phenix-online.org \\
\hline RELION 3.0.8 & (Zivanov et al., 2018) & $\begin{array}{l}\text { https://www3.mrc- } \\
\text { Imb.cam.ac.uk/relion } \\
\text { /index.php/Main_Pa } \\
\text { ge }\end{array}$ \\
\hline CTFFIND-4 & $\begin{array}{l}\text { (Rohou and Grigorieff, } \\
\text { 2015) }\end{array}$ & $\begin{array}{l}\text { https://grigoriefflab.u } \\
\text { massmed.edu/ctffind } \\
4\end{array}$ \\
\hline SCIPION & $\begin{array}{l}\text { (de la Rosa-Trevin et } \\
\text { al., 2016) }\end{array}$ & http://scipion.i2pc.es \\
\hline CURVES+ & (Blanchet et al., 2011) & $\begin{array}{l}\text { https://bisi.ibcp.fr/too } \\
\text { Is/curves_plus/index. } \\
\text { html }\end{array}$ \\
\hline MotionCor2 (included in Relion 3.0.8) & (Zheng et al., 2017) & $\begin{array}{l}\text { https://emcore.ucsf.e } \\
\text { du/ucsf-motioncor2 }\end{array}$ \\
\hline COOT 0.8 .2 & $\begin{array}{l}\text { (Emsley and Cowtan, } \\
\text { 2004) }\end{array}$ & $\begin{array}{l}\text { https://www2.mrc- } \\
\text { Imb.cam.ac.uk/perso } \\
\text { nal/pemsley/coot/ }\end{array}$ \\
\hline PyMOL & Schrödinger, LLC. & https://pymol.org/2/ \\
\hline CHIMERA & $\begin{array}{l}\text { (Pettersen et al., } \\
\text { 2004) }\end{array}$ & $\begin{array}{l}\text { https://www.cgl.ucsf. } \\
\text { edu/chimera/downlo } \\
\text { ad.html }\end{array}$ \\
\hline DYNAMICS 7.10.0.23 & Wyatt Technology & $\mathrm{N} / \mathrm{A}$ \\
\hline ResMap & $\begin{array}{l}\text { (Kucukelbir et al., } \\
2014 \text { ) }\end{array}$ & $\mathrm{N} / \mathrm{A}$ \\
\hline \multicolumn{3}{|l|}{ Other } \\
\hline Titan Krios 300 keV cryo-electron microscope & FEI / ThermoFisher & $\bar{N} / \mathrm{A}$ \\
\hline Titan Glacios 200keV cryo-electron microscope & FEI / ThermoFisher & $\mathrm{N} / \mathrm{A}$ \\
\hline PELCO easiGlow glow discharge cleaning system & Ted Pella & $\mathrm{N} / \mathrm{A}$ \\
\hline EM GP Grid Plunger & Leica & $\mathrm{N} / \mathrm{A}$ \\
\hline Quantifoil $\circledast \mathrm{R} 1.2 / 1.3200 \mathrm{Cu}$ & Quantifoil & Q42272 \\
\hline Whatman $^{\mathrm{TM}}$ filter paper, grade 1 & GE Healthcare & $1001-055$ \\
\hline
\end{tabular}




\begin{tabular}{|l|l|l|}
\hline Äkta Pure 25 L1 & GE Healthcare & 29018225 \\
\hline miniDAWN Treos & Wyatt Technology & N/A \\
\hline DynaPro NanoStar DLS & Wyatt Technology & N/A \\
\hline Optilab T-rEX & Wyatt Technology & N/A \\
\hline NanoDrop TM One UV-VIS Spectrophotometer & ThermoFishe & N/A \\
\hline Amicon® Ultra -4 10K Centrifugal filter & Merck Millipore & UFC801024 \\
\hline QuantStudio5 qPCR & Thermo Fisher & A28139 \\
\hline Superdex 200 Increase 10/300 GL & GE Healthcare & 28990944 \\
\hline Mini Dry Bath & Greiner Bio-one & 848060 \\
\hline
\end{tabular}

\section{RESOURCE AVAILABILITY}

\section{Lead Contact}

Further information and requests for resources and reagents should be directed to and will be fulfilled by the lead contact Kalyan Das (kalyan.das@kuleuven.be)

\section{Materials Availability}

All unique/stable reagents generated in this study are available from the Lead Contact with a completed Materials Transfer Agreement.

\section{Data and Code Availability}

The coordinates and density maps for y-mtRNAP PmIC and y-mtRNAP IC structures were deposited under PDB accession numbers 6YMV and 6YMW and EMBD Ids. EMD-10845 and EMD-10846, respectively. All data is available in the main text or the supplementary materials.

\section{EXPERIMENTAL MODEL AND SUBJECT DETAILS}

Not applicable. 


\section{METHODS DETAILS}

\section{Expression and purification of MTF1}

His-6 tagged $S$. cerevisiae MTF1 in pTrcHisC was used to create MTF1 mutants 1-5 using QuikChange Lightning site-directed mutagenesis kit (Agilent). MTF1 WT and mutant plasmids were transformed in E. coli strain BL21 and purified as described previously (Tang et al., 2009). Briefly, cells were grown at $37^{\circ} \mathrm{C}$ in LB media containing $100 \mu \mathrm{g} / \mathrm{ml}$ ampicillin, and after induction with $1 \mathrm{mM}$ Isopropyl $\beta$-D-1-thiogalactopyranoside (IPTG), the cells were grown at $16^{\circ} \mathrm{C}$ for $16 \mathrm{~h}$. The cell pellet was lysed by sonication in the presence of protease inhibitor and lysozyme, treated with polyethyleneimine (10\%), and ammonium sulfate (55\%) precipitated. The dissolved ammonium sulfate pellet was loaded on $5 \mathrm{ml}$ DEAE Sepharose and $5 \mathrm{ml} \mathrm{Ni-Sepharose} \mathrm{(GE} \mathrm{Healthcare} \mathrm{Life} \mathrm{Sciences)} \mathrm{connected} \mathrm{in} \mathrm{tandem.} \mathrm{The}$ DEAE Sepharose column was detached after loading and the Ni-Sepharose column washed with $50 \mathrm{ml}$ washing buffer (50 mM sodium phosphate buffer $\mathrm{pH} 8,300 \mathrm{mM} \mathrm{NaCl}, 10 \%$ glycerol, $1 \mathrm{mM}$ PMSF, $20 \mathrm{mM}$ imidazole). MTF1 protein was eluted with $70 \mathrm{ml}$ gradient of 20 $\mathrm{mM}$ to $500 \mathrm{mM}$ imidazole. The MTF1 peak eluent was collected and loaded on $2 \times 1 \mathrm{ml}$ Heparin-Sepharose columns (GE Healthcare Life Sciences), washed with $20 \mathrm{ml}$ buffer (50 mM sodium phosphate buffer $\mathrm{pH}$ 8, $150 \mathrm{mM} \mathrm{NaCl}$, 10\% glycerol, 1mM EDTA, 1 mM DTT, 1 $\mathrm{mM}$ PMSF) and eluted with $50 \mathrm{ml}$ gradient of $150 \mathrm{mM}$ to $1 \mathrm{M} \mathrm{NaCl}$. The MTF1 protein eluent was collected and concentrated using $10 \mathrm{kDa}$ MW cut-off Amicon ultra centrifugal filter and stored at $-80^{\circ} \mathrm{C}$.

\section{Expression and purification of $\Delta \mathrm{N100} \mathrm{y}$-mtRNAP}

S. cerevisiae y-mtRNAP ${ }_{\triangle \mathrm{N} 100}$ in ProEXHTb was prepared as described previously (Paratkar et al., 2011; Tang et al., 2009). Briefly, E. coli strain BL21 codon plus (RIL) transformed with ProEXHTb y-mtRNAP ${ }_{\Delta \mathrm{N} 100}$ was grown at $37^{\circ} \mathrm{C}$ in LB media containing $100 \mu \mathrm{g} / \mathrm{ml}$ ampicillin before induction with $1 \mathrm{mM}$ Isopropyl $\beta$-D-1-thiogalactopyranoside (IPTG). After 16 hours at $16^{\circ} \mathrm{C}$, the cells were harvested and lysed by sonication in the presence of protease inhibitor and lysozyme, followed by polyethyleneimine (10\%) treatment and ammonium sulfate (55\%) precipitation of the lysate. The dissolved ammonium sulfate pellet was loaded on a 5 $\mathrm{ml}$ DEAE Sepharose and a $5 \mathrm{ml} \mathrm{Ni-Sepharose} \mathrm{(GE} \mathrm{Healthcare} \mathrm{Life} \mathrm{Sciences)} \mathrm{connected} \mathrm{in}$ 
tandem. DEAE Sepharose column was detached after loading. The Ni-Sepharose column was washed with $50 \mathrm{ml}$ washing buffer (50 mM sodium phosphate buffer $\mathrm{pH} \mathrm{8,} 300 \mathrm{mM} \mathrm{NaCl}$, $10 \%$ glycerol, 1mM phenylmethylsulfonyl fluoride (PMSF), $20 \mathrm{mM}$ imidazole) and eluted using a $70 \mathrm{ml}$ gradient of $20 \mathrm{mM}$ to $500 \mathrm{mM}$ imidazole. The y-mtRNAP peak eluent was collected and loaded into $2 \times 1 \mathrm{ml}$ Heparin-Sepharose column (GE Healthcare Life Sciences). The columns were washed with $20 \mathrm{ml}$ buffer (50 mM sodium phosphate buffer $\mathrm{pH}$ 8, 150 $\mathrm{mM} \mathrm{NaCl}$, 10\% glycerol, 1 mM EDTA, 1 mM DTT, $1 \mathrm{mM}$ PMSF) and eluted with $30 \mathrm{ml}$ gradient of $150 \mathrm{mM}$ to $1 \mathrm{M} \mathrm{NaCl}$. The y-mtRNAP peak eluent was collected and treated with TEV Protease (16 hours at $4^{\circ} \mathrm{C}$ ) followed by purification by Ni-Sepharose chromatography. The flow-through was collected and concentrated using $10 \mathrm{kDa}$ MW cut-off Amicon ultra centrifugal filter and stored at $-80^{\circ} \mathrm{C}$.

\section{In vitro transcription initiation assay to measure runoff and abortive RNA synthesis}

Transcription reactions were carried out by preincubating $2 \mu \mathrm{M}$ yeast $21 S$ rRNA promoter DNA fragment or the pre-melted initiation bubble promoter with y-mtRNAP $(1 \mu \mathrm{M}), \mathrm{MTF} 1$ WT or mutant $(2 \mu \mathrm{M})$ in reaction buffer (50 mM Tris acetate pH 7.5, $100 \mathrm{mM}$ potassium glutamate, $10 \mathrm{mM}$ magnesium acetate, $1 \mathrm{mM}$ DTT, $0.01 \%$ Tween 20 ) at $25^{\circ} \mathrm{C}$, and initiating RNA synthesis by adding NTPs (100 $\mu$ M each of ATP, UTP, GTP, and 1.25 mM 3'-dCTP spiked with $\alpha$-32P-GTP. Reactions were terminated after 15 min using 125 mM EDTA and formamide dye mixture (98\% formamide, $0.025 \%$ bromophenol blue, 10 mM EDTA), and RNA products were resolved on a $24 \%$ polyacrylamide, $4 \mathrm{M}$ urea gel. The gel image was analyzed using ImageQuant to calculate the amount of runoff RNA products.

\section{2-aminopurine assays for DNA melting and initiating NTP binding}

The yeast $21 S$ rRNA promoter DNA labeled with 2-aminopurine (2AP) at position -4 in the non-template strand (200 nM) was mixed with y-mtRNAP (400 nM) and MTF1 WT or mutants (400 nM) and the fluorescence intensity (excitation at $315 \mathrm{~nm}$ and emission 380 $\mathrm{nm}$ ) was recorded in a FluoroMax spectrometer (Hiroba Scientific) at $25^{\circ} \mathrm{C}$. To measure the $\mathrm{K}_{\mathrm{d}}$ of initiating NTPs, the promoter DNA was labeled with 2AP at position -1 in the template strand. Increasing concentrations of initiating nucleotides (ATP+GTP) were added to a complex of 2AP promoter DNA (200 nM), y-mtRNAP (400 nM), and MTF1 WT or mutants 
(400 nM) and fluorescence emission (380 $\mathrm{nm}$ after excitation at $315 \mathrm{~nm}$ ) was recorded. The titration data estimated the cumulative $K_{d}$ of the initiating nucleotides when fit to a hyperbolic equation $\left(\mathrm{Fc}=\mathrm{Fm}^{*}[\mathrm{~N}] / \mathrm{Kd}_{\mathrm{d}}+[\mathrm{N}]\right.$, where $\mathrm{Fc}$ is the fluorescence intensity at a given concentration of $\mathrm{ATP}+\mathrm{GTP}(\mathrm{N})$, and Fm is the maximum fluorescence change).

\section{Ultrafiltration assay to monitor y-mtRNAP - MTF1 interaction}

An equimolar complex of y-mtRNAP and MTF1 (2 $\mu \mathrm{M}$ each) were mixed in $50 \mathrm{mM}$ Tris acetate, $\mathrm{pH}$ 7.5, $100 \mathrm{mM}$ potassium glutamate, $10 \mathrm{mM}$ magnesium acetate, $5 \mathrm{mM}$ fresh DTT, $0.01 \%$ protein-grade Tween $20,5 \%$ glycerol in a final volume of $500 \mu \mathrm{L}$. The mixture was incubated at $25^{\circ} \mathrm{C}$ for 15 min before filtering through a $100 \mathrm{kDa} \mathrm{MW}$ cut-off Microcon centrifugal filter unit until the volume of the first retentate was about $50 \mu \mathrm{l}(1 / 10$ of initial mixture). The retentate diluted to $500 \mu \mathrm{l}$ with the above buffer and filtered again. This washing step was repeated, and a sample was taken after washes. Samples consisting of initial protein complex, first retentate, filtrate and retentate samples were collected and run on a $4-20 \%$ SDS-PAGE gel (Figure S3F).

\section{Assembly and characterization of partially-melted initiation complex (PmIC) and initiation complex (IC)}

The y-mtRNAP PmIC was prepared by incubating y-mtRNAP ${ }_{\triangle \mathrm{N} 100}, \mathrm{MTF} 1$, and promotor DNA in a molar ratio 1:1.2:1.2 for two hours at $4^{\circ} \mathrm{C} ; \Delta \mathrm{N} 100$ represent the y-mtRNAP construct in which the first $100 \mathrm{~N}$-terminal amino acids are deleted. The complex at a starting concentration of $6 \mathrm{mg} / \mathrm{ml}$ in buffer A (50mM Bis-tris propane $\mathrm{pH} 7.0,100 \mathrm{mM} \mathrm{NaCl}, 5 \mathrm{mM}$ MgCl2, 1mM EDTA, 2mM DTT) was purified by size-exclusion chromatography. A Superdex 200 Increase 10/300 GL column was mounted on a GE AKTA Pure 25 FPLC in-line with Wyatt Technology multi-angle light-scattering (MALS) device mini-DAWN, differential refractive index (dRI) measuring device Optilab, and dynamic light scattering (DLS) device DynaPro Nanostar. The purification was carried out at $6^{\circ} \mathrm{C}$ temperature. The purified y-mtRNAP complex sample at a concentration of $2.7 \mathrm{mg} / \mathrm{ml}$ in buffer containing $50 \mathrm{mM}$ Bis-tris propane, $100 \mathrm{mM} \mathrm{NaCl}, 5 \mathrm{mM} \mathrm{MgCl}$, 1 mM EDTA, 2 mM DTT at pH 7.0 was aliquoted and stored at $80^{\circ} \mathrm{C}$. The transcription initiation complex (y-mtRNAP IC) was prepared by incubating $\mathrm{y}$ - 
mtRNAP PmIC, 2-mer RNA, and non-hydrolysable UTP $\alpha$ S at a molar ratio of 1:6:50 for two hours over ice.

The light-scattering measurements (Minton, 2016) reliably confirmed the formation of homogenous complex with expected molecular weight (Figure S1B-E). The same protocol was used for purifying y-mtRNAP PmIC containing full-length $y$-mtRNAP. The DLS experiments in stand-alone cuvette mode using Nanostar did not show any significant difference of hydrodynamic radius or polydispersity between y-mtRNAP PmIC and ymtRNAP IC samples. For the DLS experiments, $8 \mu \mathrm{L}$ of the $y$-mtRNAP IC or $y$-mtRNAP PmIC complex at a concentration of $\sim 0.5 \mathrm{mg} / \mathrm{ml}$ was loaded into a Wyatt disposable cuvette. The sample cuvette was placed in the sample chamber maintained at $4^{\circ} \mathrm{C}$. Thirty acquisitions were taken for each sample, and the data were analyzed using Dynamics (Version 7.10.0.23) software (Wyatt Technology). Samples were always characterized by the DLS experiments prior to preparation of cryo-EM grids to ensure consistency and reproducibility.

A high-throughput thermal shift assay (Huynh and Partch, 2015) showed higher stability of y-mtRNAP PmIC compared to y-mtRNAP-only sample (Figure S1F). The thermal shift experiment was conducted in quadruplicate using a ThermoFisher QuantStudio5 qPCR setup and Protein Thermal Shift ${ }^{\mathrm{TM}}$ dye (Applied Biosystems, Cat. NO. 4461146) in 96 well format. Each well had $5 \mu \mathrm{g}$ of protein in a volume of $20 \mu \mathrm{l}$ and $1 \mathrm{x}$ dye. The temperature was ramped from $4^{\circ} \mathrm{C}$ to $95^{\circ} \mathrm{C}$ at a rate of $3^{\circ} \mathrm{C} / \mathrm{min}$. The fluorescence from the dye was measured at $623 \pm 14 \mathrm{~nm}$ wavelength while using an excitation wavelength of $580 \pm 10 \mathrm{~nm}$. The data were analyzed using Protein Thermal Shift 1.3 software. The $\mathrm{T}_{\mathrm{m}}$ of $\mathrm{y}$-mtRNAP PmIC is $47^{\circ} \mathrm{C}$ compared to $42^{\circ} \mathrm{C}$ for $\Delta 100$-only sample, showing higher stability of the complex.

\section{Cryo-EM data collection and processing}

The vitreous grids of $y$-mtRNAP IC were prepared on Quantifoil R 1.2/1.3 holey carbon grids using a Leica EM GP. The grids were glow-discharged for 30s at $10 \mathrm{~mA}$ current with the chamber pressure set at $0.30 \mathrm{mBar}$ (PELCO easiGlow; Ted Pella). Prior to glow-discharge, the grids were placed over a filter paper soaked with chloroform for two hours and dried overnight. The glowdischarged grids were mounted in the sample chamber of a Leica EM GP at $8^{\circ} \mathrm{C}$ and $95 \%$ relative humidity, blotted, and plunge-frozen in liquid ethane at temperature $-172^{\circ} \mathrm{C}$. The frozen grids were 
tested on a JEM-1400 transmission electron microscope (TEM) at VUB Brussels, and the grid preparation conditions were optimized in cycles. The final optimized grids were reproducibly prepared using $5 \mu \mathrm{L}$ of sample at a concentration of $1 \mathrm{mg} / \mathrm{ml}$ (in $50 \mathrm{mM}$ Bis-tris propane, $100 \mathrm{mM}$ $\mathrm{NaCl}, 5 \mathrm{mM} \mathrm{MgCl}$, $1 \mathrm{mM}$ EDTA, $2 \mathrm{mM}$ DTT at $\mathrm{pH}$ 7.0) spotted on Quantifoil R 1.2/1.3 holey carbon grids, incubated on the grid for 30 seconds, and back blotted for 12 - 14 seconds using two pieces of Whatman ${ }^{\circledR}$ Grade 1 filter paper. The grids of $y$-mtRNAP PmIC were prepared using the conditions used for $y$-mtRNAP IC grids.

A preliminary dataset collected from a cryo-EM grid of y-mtRNAP IC using a $200 \mathrm{kV}$ Glacios/Falcon-3EC setup revealed the existence of IC and PmIC states. High-resolution data sets for y-mtRNAP IC and y-mtRNAP PmIC were collected at ESRF-Grenoble CM01 facility using a $300 \mathrm{kV}$ Titan KRIOS TEM equipped with a Gatan K2 Summit direct electron detector and a Gatan energy filter (Kandiah et al., 2019). The data collection for y-mtRNAP PmIC was automated using EPU version 2.5 (ThermoFisher). Electron movies were collected in the counting mode at a nominal magnification of 165,000x. The total exposure time was 6 seconds with a total dose of $65 \mathrm{e}^{-} / \AA^{2}$ and the movies were recorded as gain corrected MRC files. Images for y-mtRNAP IC sample were collected using EPU software version 2.6.1 (ThermoFisher) in the counting mode again at a nominal magnification of 165,000x yielding a pixel size of $0.827 \AA$. The exposure time was 5 seconds for each movie, accumulating to a total dosage of $61 \mathrm{e}^{-} / \AA^{2}$. The beam-image shift was applied during data collection to increase data throughput. Movies were recorded as compressed MRC files. For both data collections, the energy filter was used with a slit width of $20 \mathrm{eV}$. The data collection parameters are listed in Table 1.

All frames in individual movies were aligned using MotionCor2 (Zheng et al., 2017) as implemented in Scipion (de la Rosa-Trevin et al., 2016) and contrast-transfer-function (CTF) estimations were performed using CTFFIND-4 (Rohou and Grigorieff, 2015). A reference set of thirteen 2D classes were obtained earlier from 18,648 particles from Glacios data picked using Xmipp picker. The particles were classified into thirteen 2D classes using Relion 3.0.8 (Zivanov et al., 2018), and the set of thirteen classes was used as the template for picking the particles from high-resolution y-mtRNAP PmIC and y-mtRNAP IC datasets using Autopick routine of Relion 3.0.8. A schematic representation of the data processing is shown in Figure 
S2A-B. For the final map calculations, 48 out of 60 frames for y-mtRNAP PmIC and 42 out of 50 frames for $y$-mtRNAP IC were re-aligned using the Relion-implemented MotionCor2. The particles are re-extracted, fitting of CTF parameter and per-particle defocus were applied using Relion 3.0.8 prior to the final map calculation using Refine3D, and the maps were Bsharpened using the Postprocessing routine in Relion. The final maps were calculated at $3.1 \AA$ and $3.7 \AA$, respectively, for $y$-mtRNAP PmIC and y-mtRNAP IC structures. The local resolutions of the maps were calculated using ResMap (Kucukelbir et al., 2014).

\section{Model building}

The $3.1 \AA$ A map for y-mtRNAP PmIC complex was used to build the atomic model of ymtRNAP. The structures of h-mtRNAP in h-mtRNAP IC (PDB Id. 6EQR) and of T7 RNAP in T7 RNAP IC (PDB Id. 1QLN) were used as references while building the model for y-mtRNAP. The crystal structure of MTF1 (PDB Id. 1I4W) was modeled into its part of the density. The model building was done manually using COOT (Emsley and Cowtan, 2004). The real-space fitting of the model to the density map was carried out using Phenix 1.16 (Liebschner et al., 2019). In the final $y$-mtRNAP PmIC structure, MTF1 is traced from $S 2$ - Q336, and y-mtRNAP is traced from I386 till the end residue S1351, with missing stretches 559 - 588, 1024 - 1049, 1281 - 1300, 1315 - 1317. The final y-mtRNAP IC structure has MTF1 traced from S2 - G341 (end residue) and y-mtRNAP from I386 to S1351 with disordered pieces 1281 - 1300, 1315 - 1317; the fingers subdomain, which is partially disordered in PmIC structure, is completely traced in IC structure. The structure figures were generated using PyMOL(https://pymol.org/2/) and Chimera (Pettersen et al., 2004). 

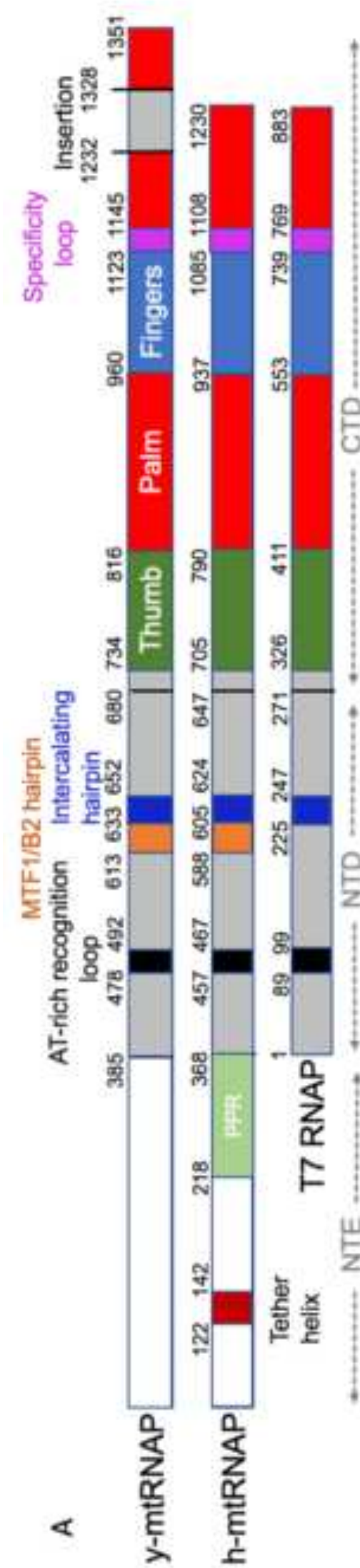

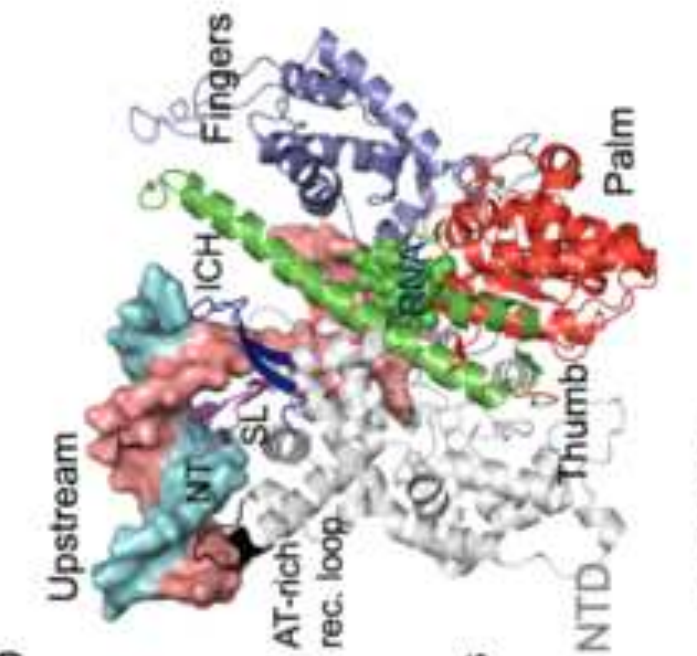

$\frac{\substack{s \\ \frac{1}{\alpha}}}{F}$

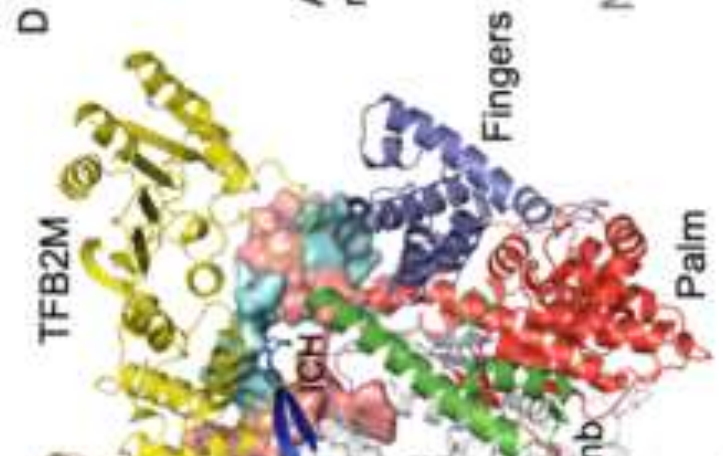

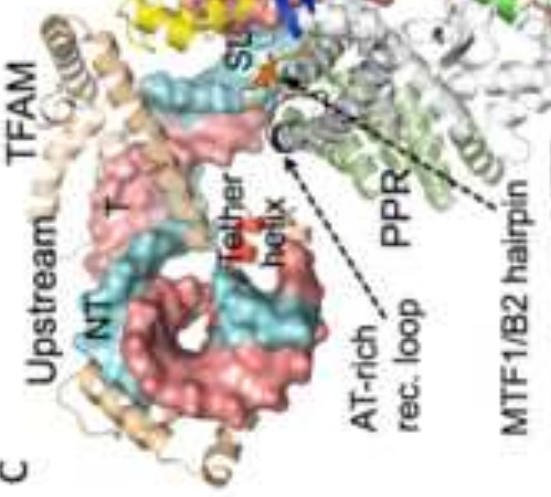

$\sum_{\frac{1}{2}}^{\frac{0}{2}}$

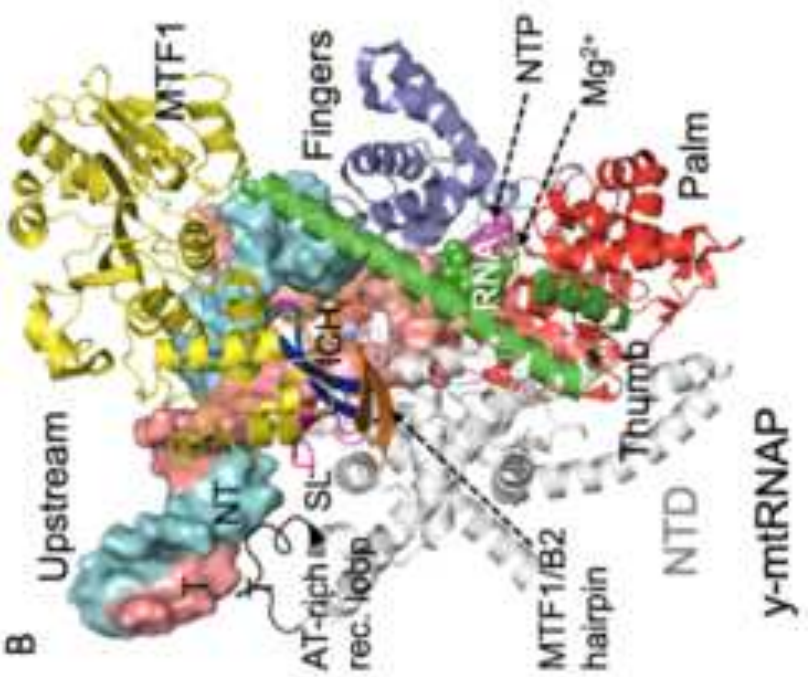



A -21
$-4$
$+2$
$+12$
$5^{\prime}$-CGAATAAGTATTGATATAAGTAATAGATAATGC 3'-GCTTATTCATAACTATA
ATCTATTACG
(NT)
( T) ACAGCC
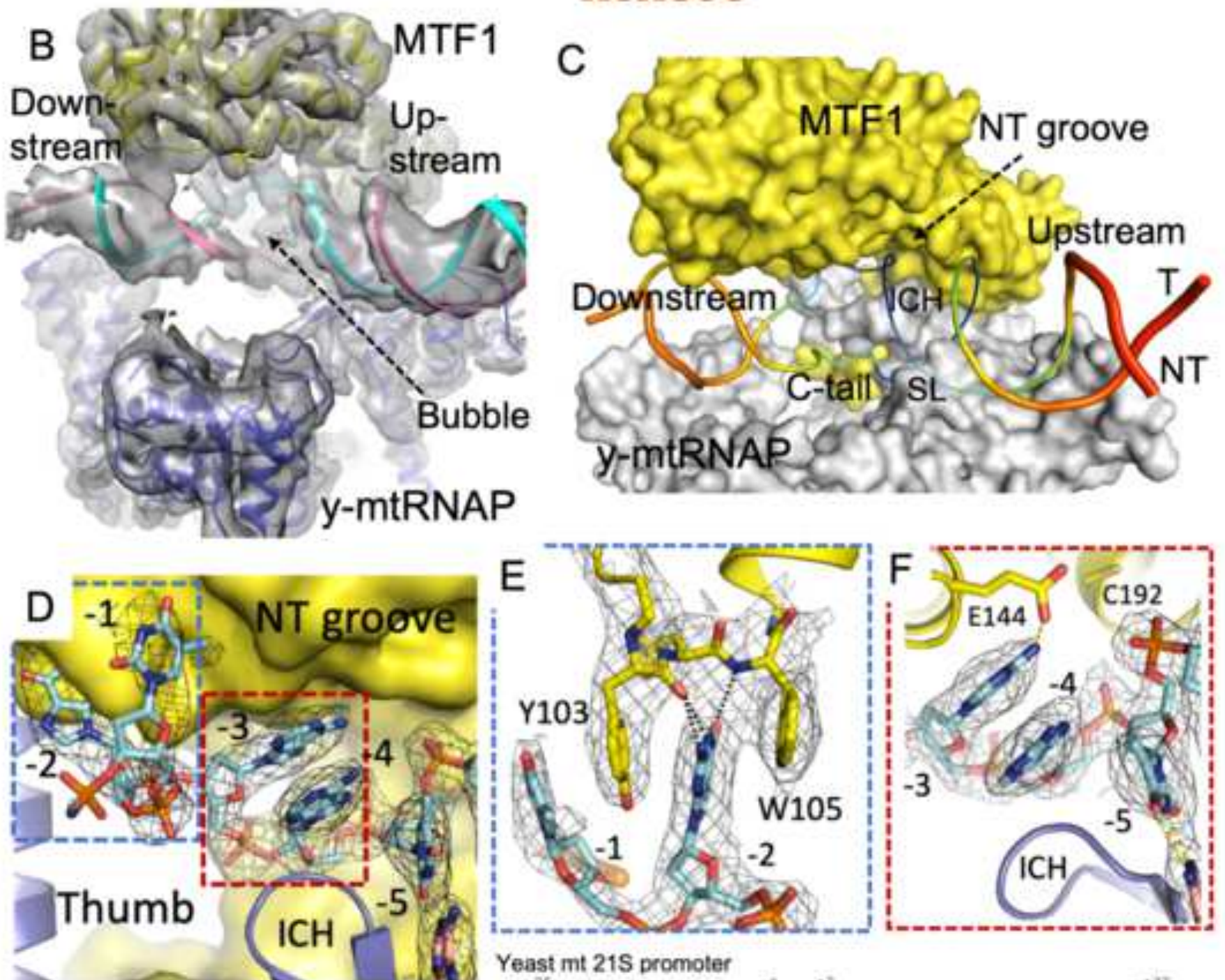

Yeast $\mathrm{mt} 21 \mathrm{~S}$ promoter

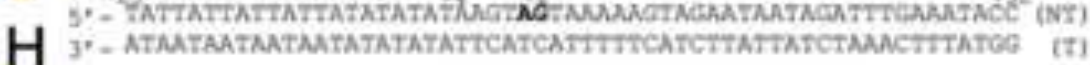
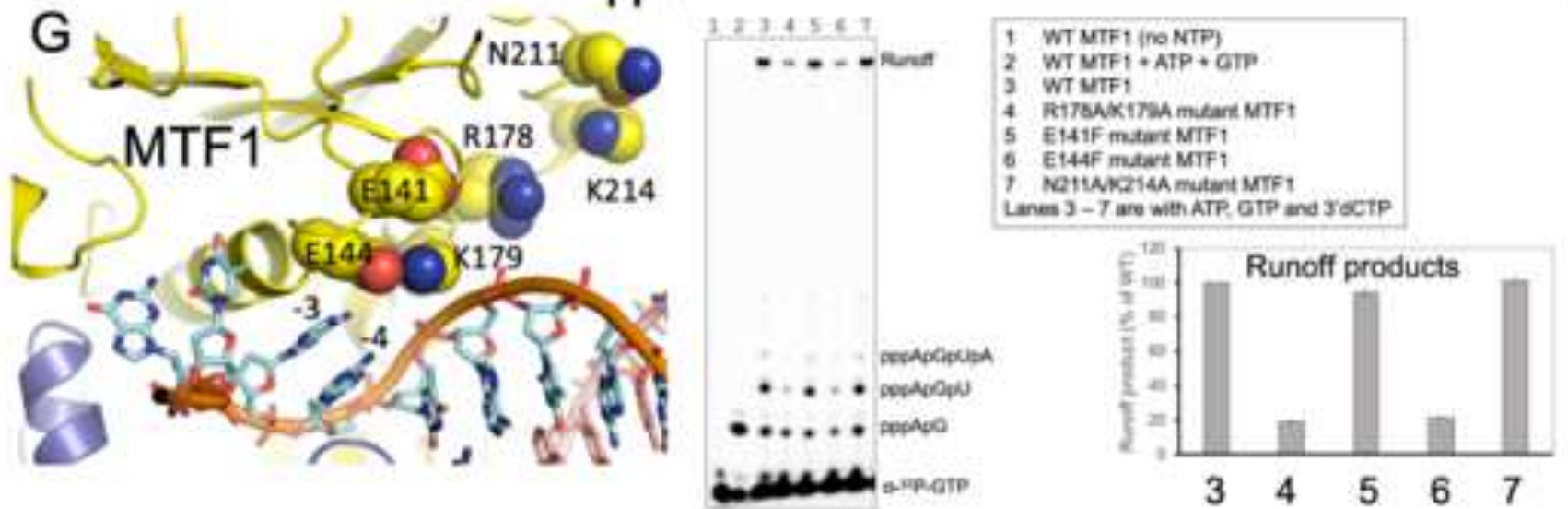
Figure 3
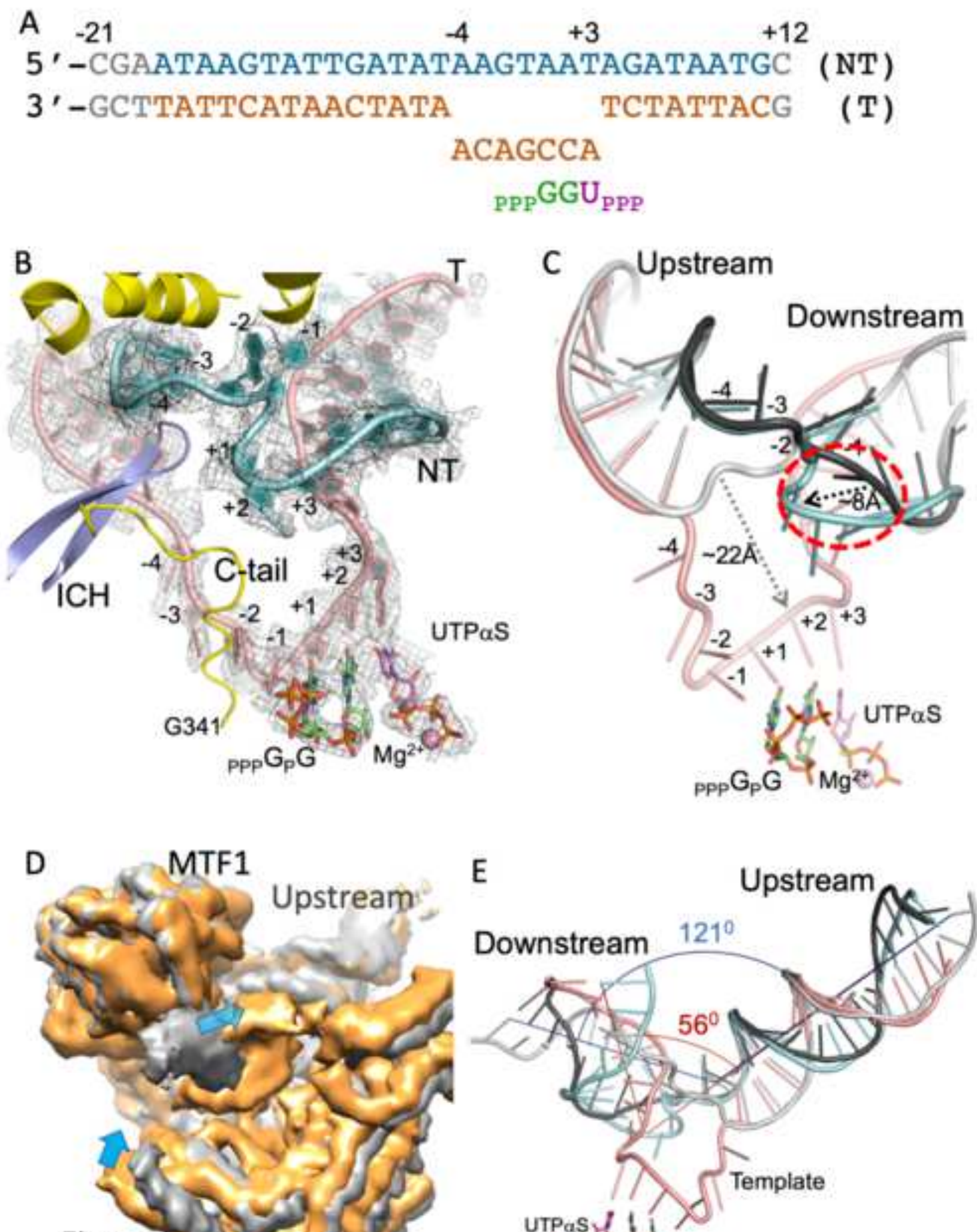

Fingers

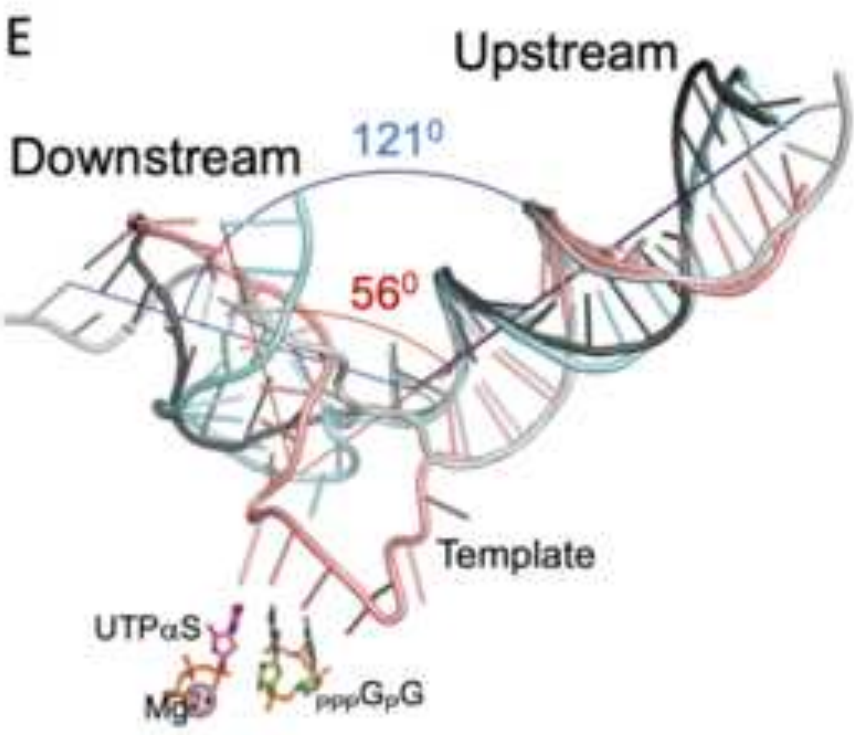



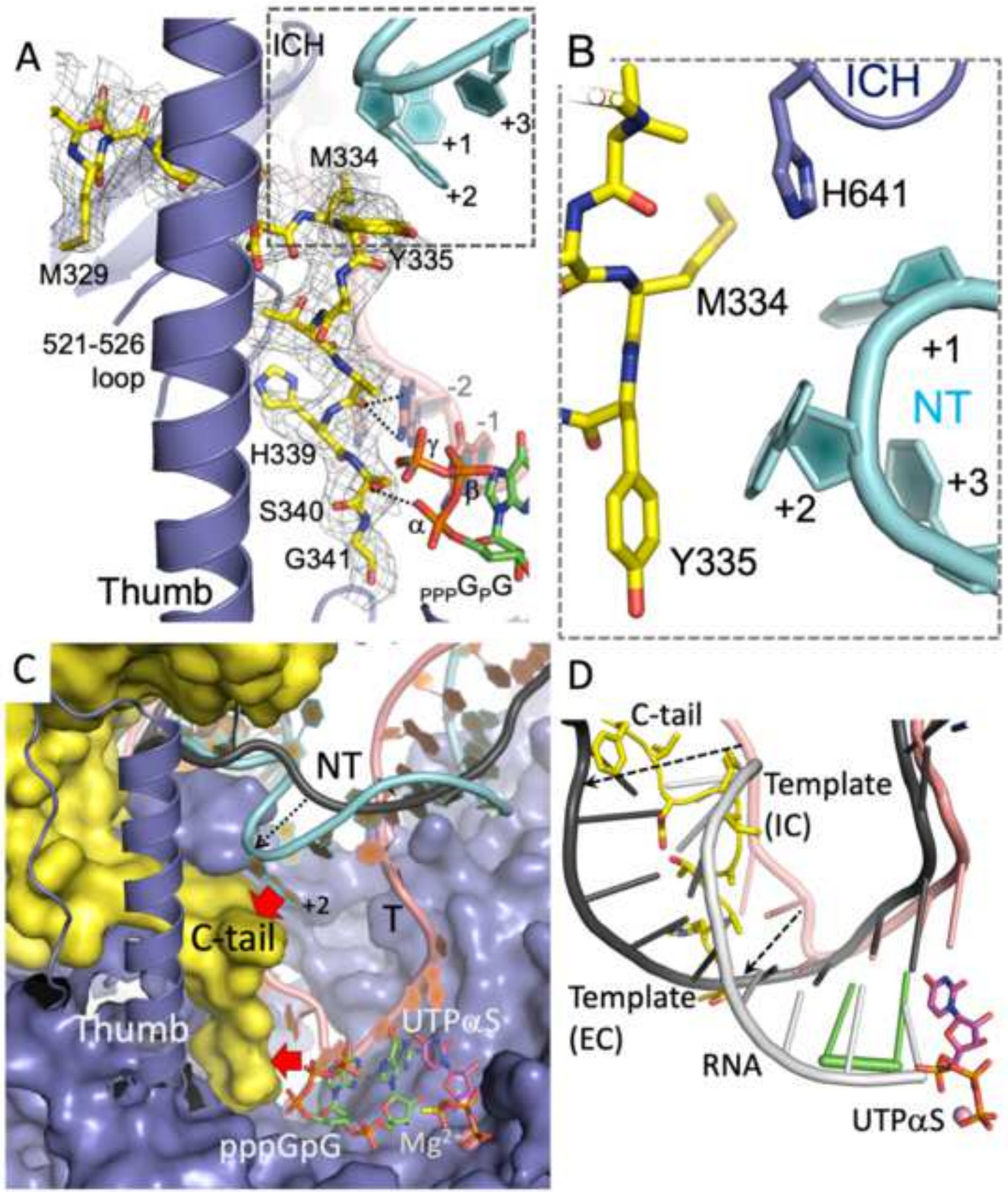

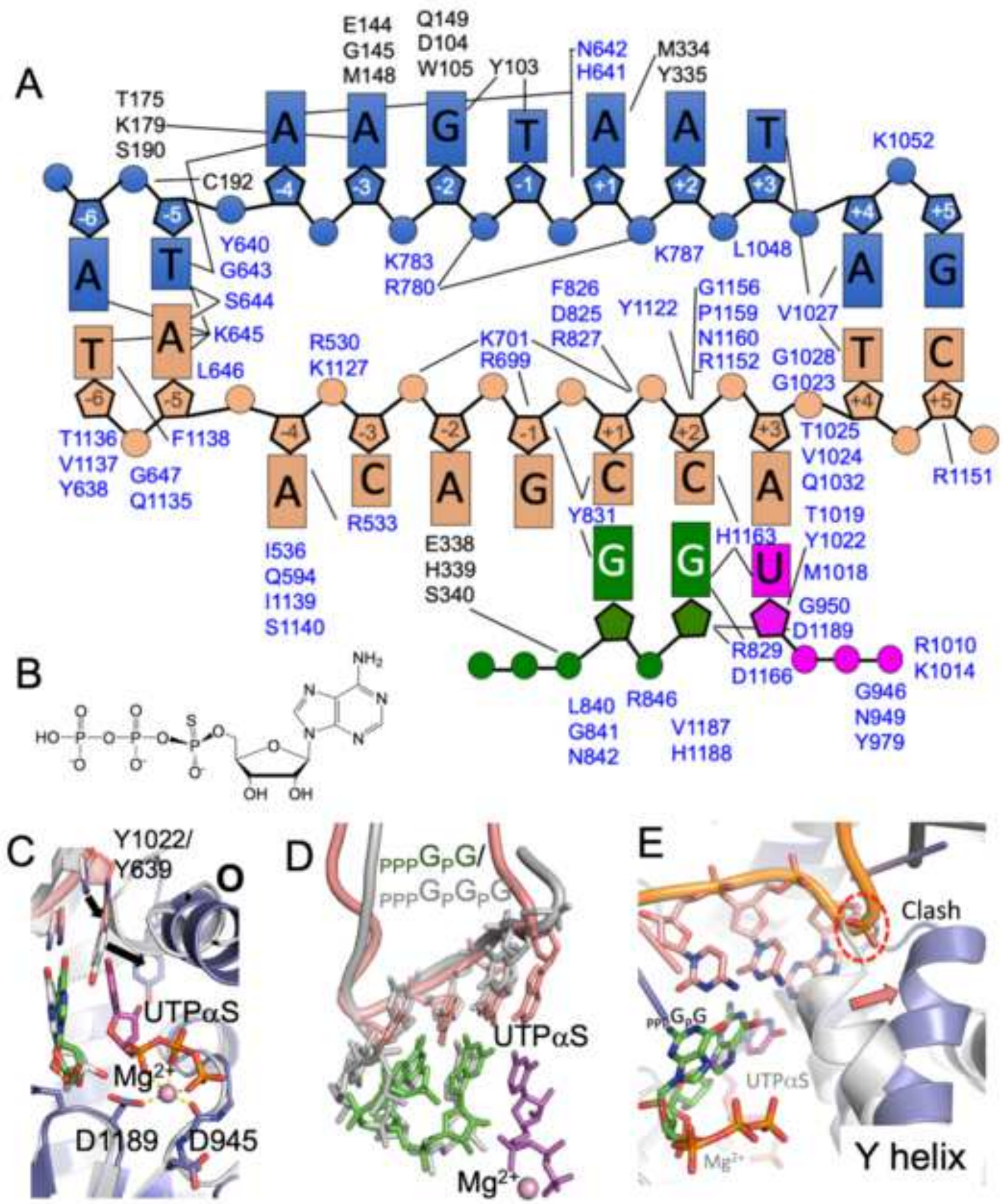

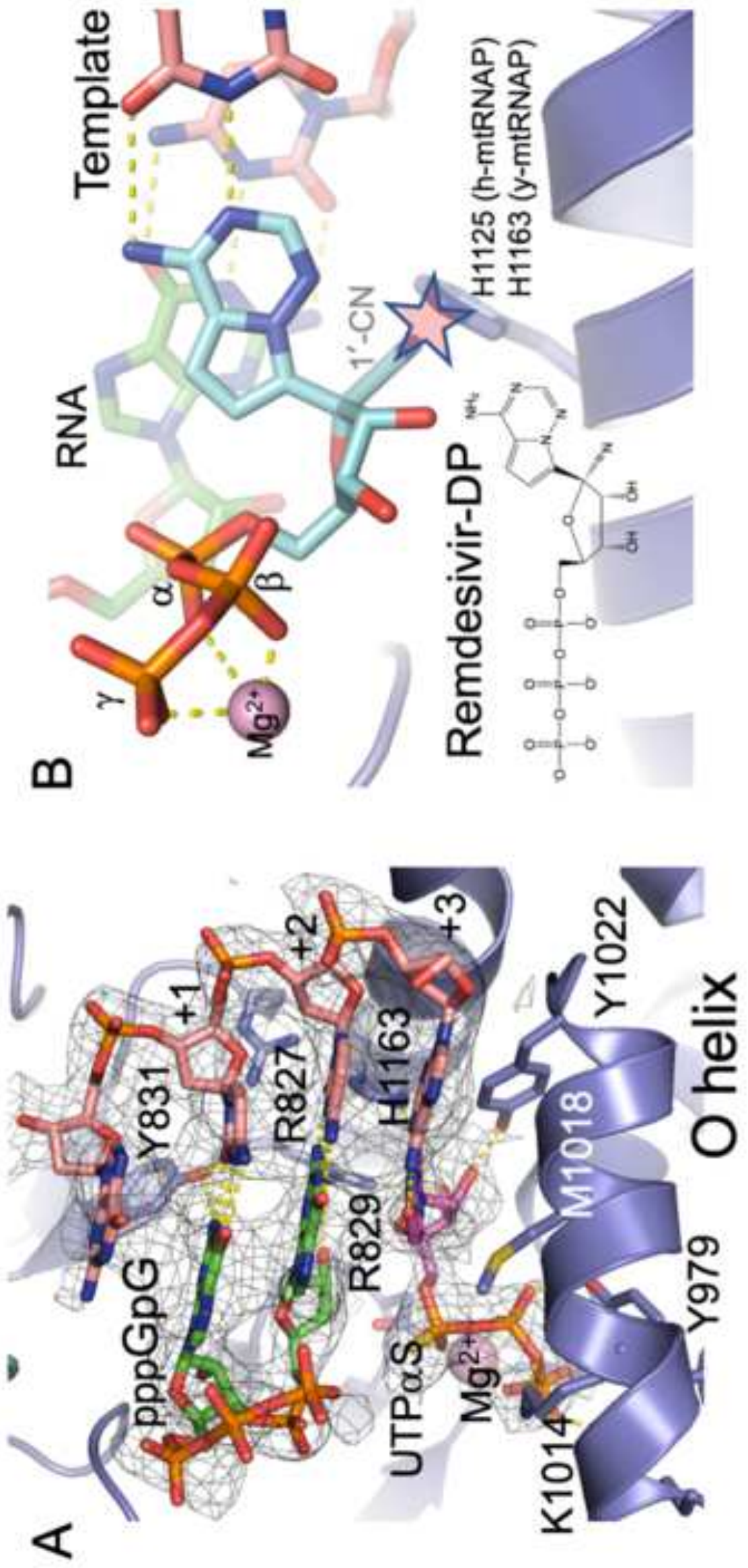
Table 1. Single particle cryo-EM data and structure analysis statistics.

\begin{tabular}{|c|c|c|}
\hline Structure & $\begin{array}{l}\text { PmIC (y-mtRNAP; } \\
\text { MTF1:dsDNA) }\end{array}$ & $\begin{array}{l}\text { IC (y-mtRNAP:MTF1: } \\
\text { dsDNA:RNA:UTP } \alpha S)\end{array}$ \\
\hline PDB Id/EMBD Id & 6YMV/EMD-10845 & 6YMW/EMD-10846 \\
\hline \multicolumn{3}{|l|}{ Data collection } \\
\hline Grid type & Quantifoil R1.2/1.3 & Quantifoil R1.2/1.3 \\
\hline Number of grids & 1 & 1 \\
\hline Microscope/detector & Titan Krios/Gatan K2 & Titan Krios/Gatan K2 \\
\hline Voltage $(\mathrm{kV})$ & 300 & 300 \\
\hline Magnification & $165,000 x$ & $165,000 x$ \\
\hline Recording mode & Counting & Counting \\
\hline Dose (e-/ $\AA^{2} /$ frame $)$ & 1.09 & 1.22 \\
\hline Total dose $\left(\mathrm{e} / \AA^{2}\right)$ & 65 & 61 \\
\hline Number of frames/movies & 60 & 50 \\
\hline Total exposure time (sec) & 6 & 5 \\
\hline Pixel size $(\AA)$ & 0.827 & 0.827 \\
\hline Defocus range $(\AA)$ & -7000 to -22000 & -7000 to -22000 \\
\hline \multicolumn{3}{|l|}{ Data processing } \\
\hline Number of micrographs used & 1,302 & 2,684 \\
\hline Number of particles picked & 492,574 & 775,698 \\
\hline Number of particles after 2D & 346,015 & 473,263 \\
\hline Particles used for final map & 152,504 & 62,807 \\
\hline Map resolution (FSC $0.143 ; \AA$ ) & 3.1 & 3.7 \\
\hline Map sharpening $B$ factor $\left(\AA^{2}\right)$ & -114.98 & -163.95 \\
\hline \multicolumn{3}{|l|}{ Model fitting } \\
\hline Experimental map/model correlation & 0.84 & 0.83 \\
\hline Total number of atoms & 10,944 & 11,320 \\
\hline \multicolumn{3}{|l|}{ Average $\mathrm{B}$ factor $\left(\AA^{2}\right)$} \\
\hline Protein atoms & 47 & 48 \\
\hline Nucleic acid and NTP & 137 & 111 \\
\hline Clash score & 13 & 15 \\
\hline $\begin{array}{l}\text { Ramachandran plot; favored/outlier } \\
(\%)\end{array}$ & $95.79 / 0.00$ & $94.69 / 0.00$ \\
\hline Rotamer outlier $(\%)$ & 0.9 & 0.6 \\
\hline $\begin{array}{l}\text { RMSD bond length }(\AA) / \text { bond angle } \\
\left({ }^{\circ}\right)\end{array}$ & $0.007 / 0.728$ & $0.008 / 0.81$ \\
\hline
\end{tabular}




\title{
SUPPLEMENTAL INFORMATION
}

\section{Cryo-EM structures reveal transcription initiation steps by yeast mitochondrial RNA polymerase}

\author{
Brent De Wijngaert ${ }^{1}$, Shemaila Sultana ${ }^{2}$, Anupam Singh ${ }^{2}$, Chhaya Dharia ${ }^{2}$, Hans \\ Vanbuel $^{1}$, Jiayu Shen ${ }^{2}$, Daniel Vasilchuk ${ }^{2}$, Sergio E. Martinez ${ }^{1}$, Eaazhisai Kandiah ${ }^{3}$, \\ Smita S. Patel ${ }^{2,}$, Kalyan Das ${ }^{1,4, *}$ \\ ${ }^{1}$ Rega Institute for Medical Research, The Department of Microbiology, Immunology and \\ Transplantation, KU Leuven, 3000 Leuven, Belgium \\ ${ }^{2}$ Department of Biochemistry and Molecular Biology, Robert Wood Johnson Medical School, \\ Rutgers University, Piscataway, NJ 08854, USA \\ ${ }^{3}$ European Synchrotron Radiation Facility, 71, avenue des Martyrs, 38043 Grenoble, France \\ ${ }^{4}$ Lead Contact \\ *Correspondence to: kalyan.das@kuleuven.be and patelss@,rwjms.rutgers.edu
}



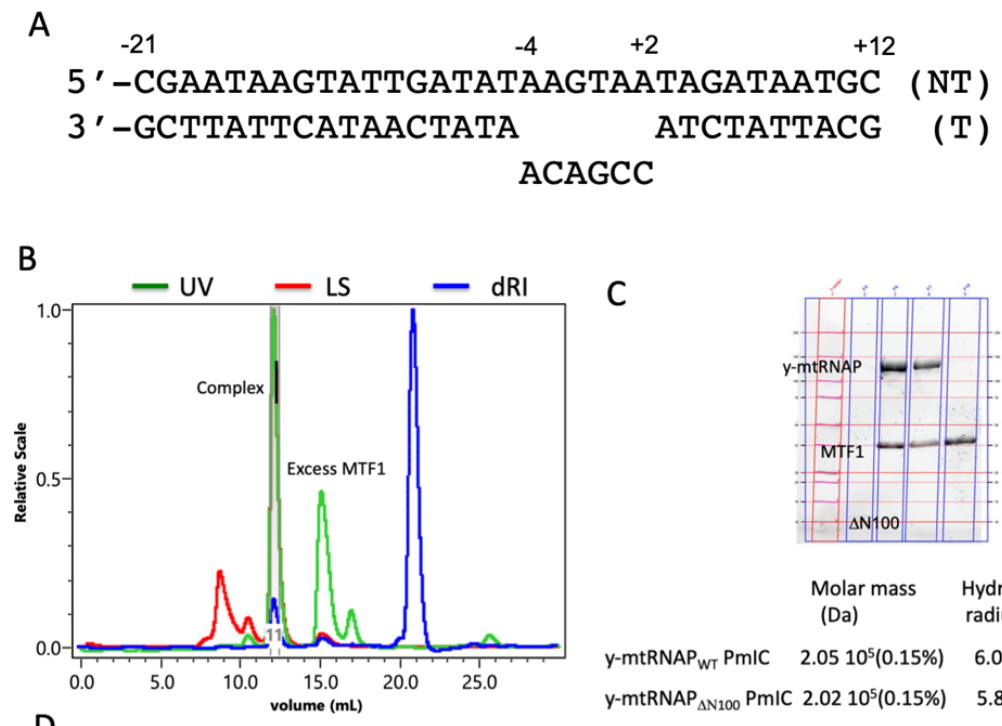

C
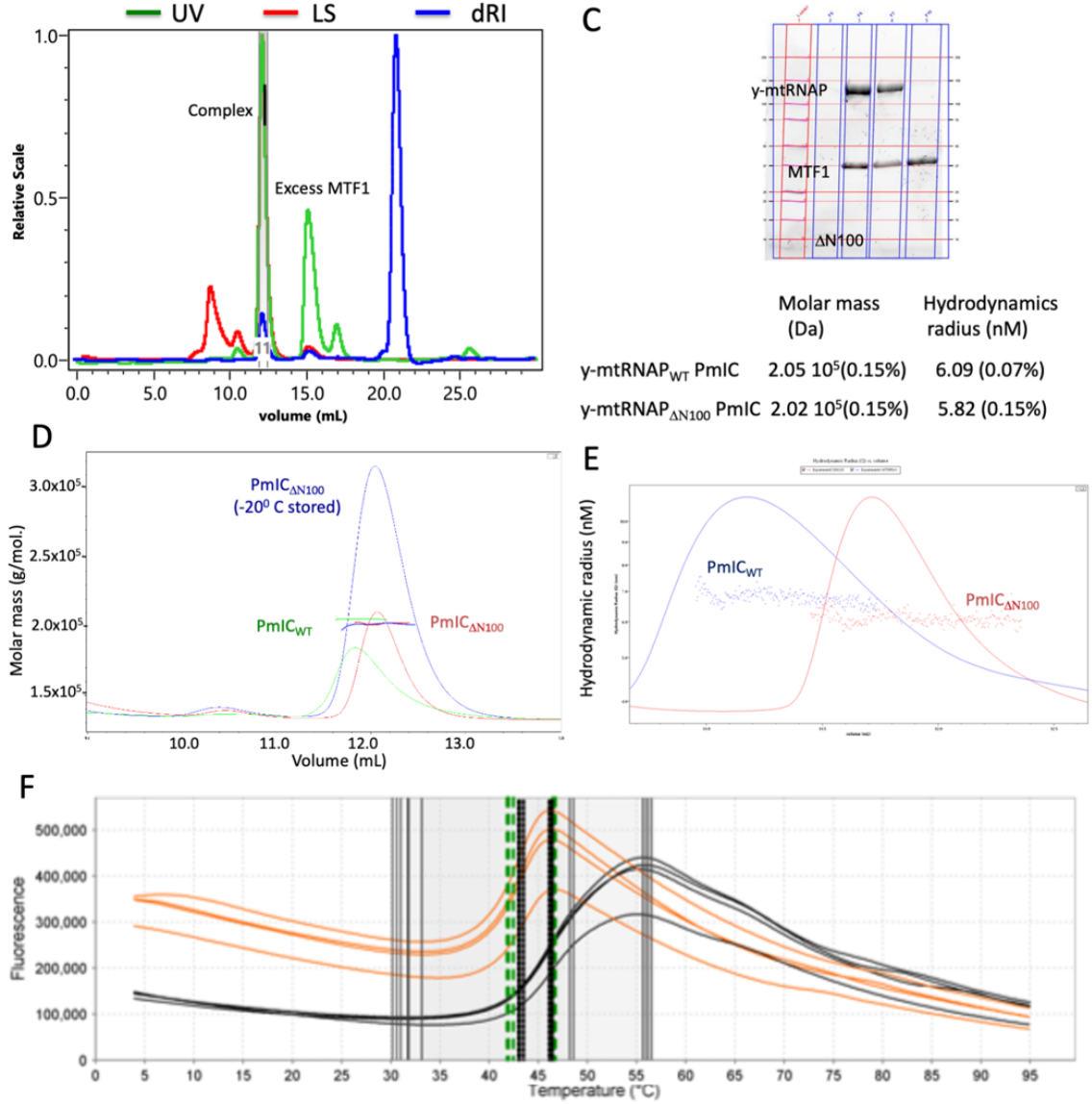

$y$-mtRNAP ${ }_{\triangle N 100}$ y-mtRNAP ${ }_{\triangle N 100}$ PmIC

Figure S1: Biophysical characterization of y-mtPmIC. (A) UV, MALS/DLS, and dRI profile of the $y$ - mtRNAP PmIC on gel-filtration. (B) SDS-PAGE gel of the $y$-mtRNAP PmIC fraction (first two lanes from left) and excess MTF1 (right); calculated molar mass in Dalton (Da) and hydrodynamic radius (nanometer) of $y$-mtRNAP PmIC measured by the MALS and DLS setups. (C) Comparison of molar mass of $y$-mtRNAP PmIC of full-length y-mtRNAP ( $y$-mtRNAP ${ }_{w T}$ PmIC) and of $\mathrm{N}$-terminal 100 amino-acid deletion mutant $y$-mtRNAP $\left(y-m t R N A P{ }_{\triangle N 100} P m I C\right) . ~-80^{\circ} \mathrm{C}$ freeze-thaw did not damage $\mathrm{y}-\mathrm{mtPmIC}_{\Delta \mathrm{N} 100}$ and the sample could be stored in small aliquots for subsequent uses. (D) Hydrodynamic radius measurements of y-mtPmIC $\mathrm{WT}_{\mathrm{WT}} \mathrm{vs}$. y-mtPmIC $\mathrm{C}_{\Delta \mathrm{N} 100}$. (E) Protein thermal shift assay profile of $y-\mathrm{mtPmIC}_{\triangle \mathrm{N} 100}$ (black curve) vs. $y$-mtRNAP ${ }_{\Delta \mathrm{N} 100}$ (orange curve) in quadruplicate measurements show higher stability of the complex. 


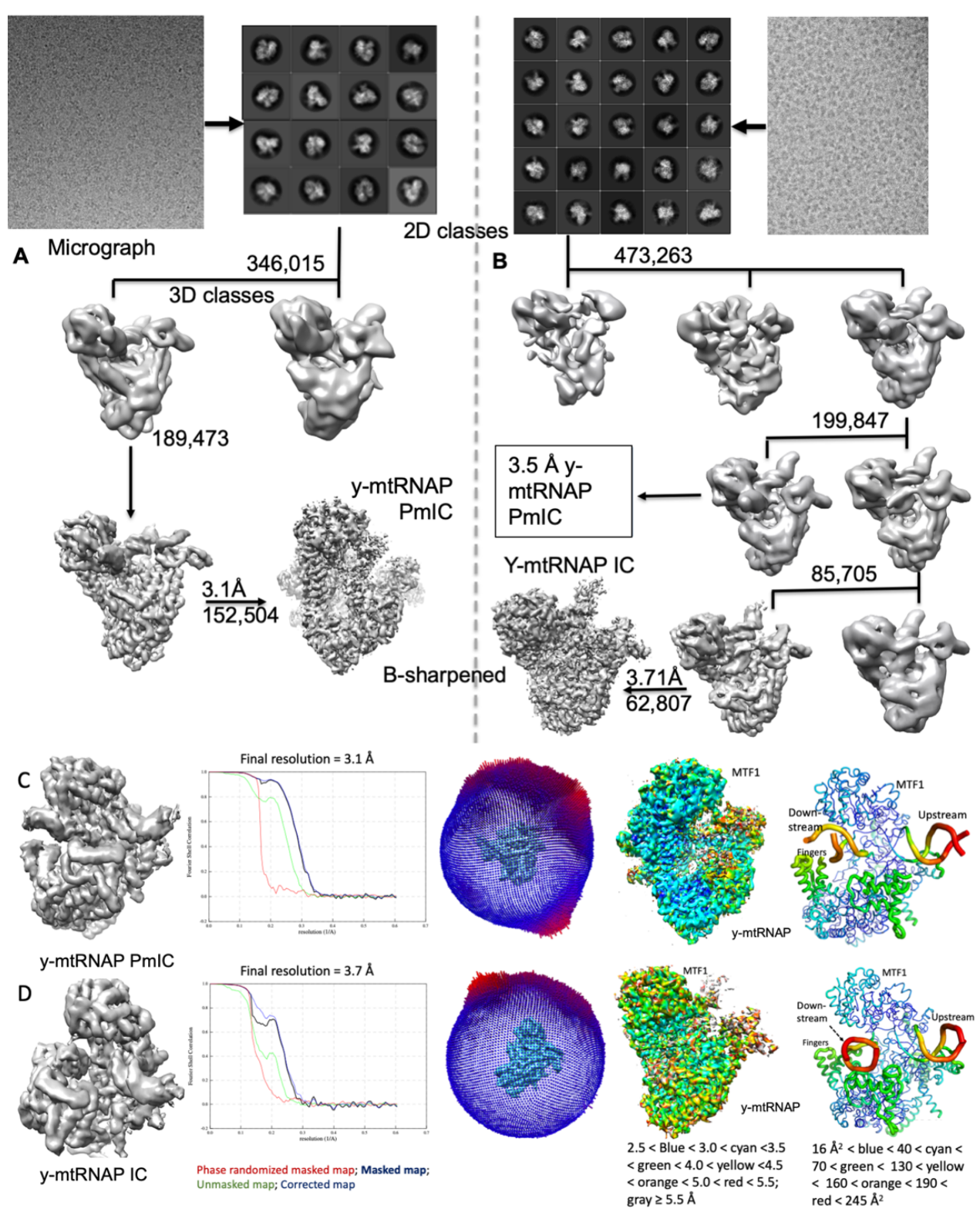

Figure S2. Cryo-EM data processing and density maps. (A) Processing of y-mtRNAP PmIC micrographs and the roadmap for obtaining the final map was calculated at $3.1 \AA$ resolution. The number of particles used at each step are listed. (B) Processing of $y$-mtRNAP IC micrographs and the roadmap for obtaining the final $y$-mtRNAP IC map was calculated at $3.7 \AA$ resolution. The $y$-mtRNAP PmIC particles grouped in the step 2 of 3D classification were processed to generate a density map at $3.5 \AA$ resolution (not shown here) and map was confirmed to represent a state which is the same as the $3.1 \AA$ y-mtRNAP PmIC map. For y-mtRNAP PmIC (C) and y-mtRNAP IC (D) structures, final density map from 3D refinement, FSC curve, angular distribution of the final set of particles, B-sharpened map color coded with estimated local resolution, and the Bfactor puffy representation of the final model (blue < cyan < green < yellow < orange $<$ red colors represent low to high B-factors). 
A

C-terminal domain (CTD)

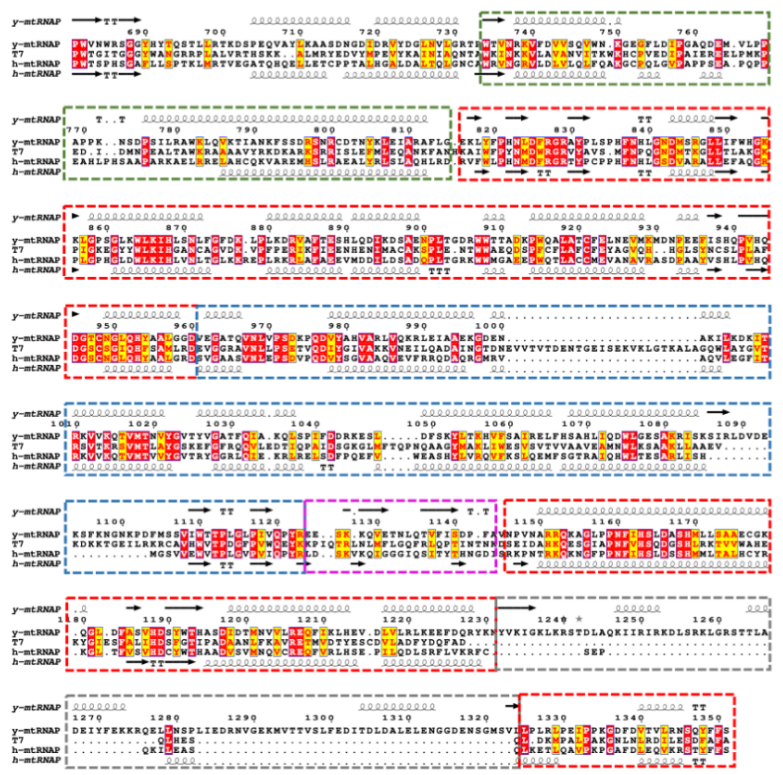

B

$\mathrm{N}$-terminal domain (NTD)
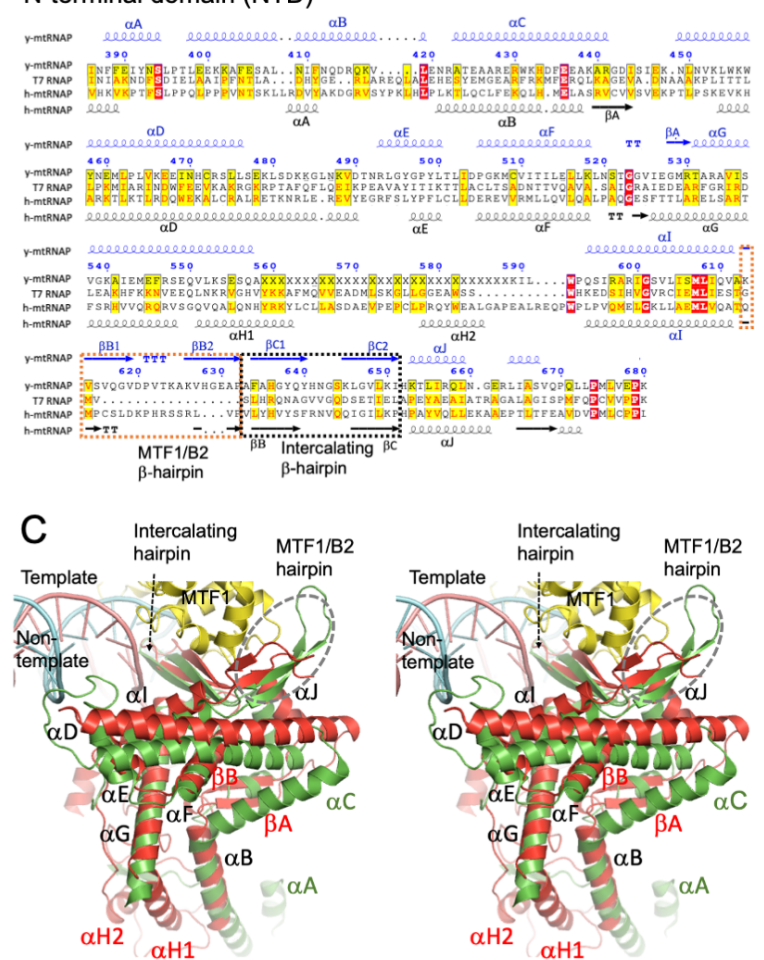

Figure S3. Amino acid sequence comparison of single-subunit RNA polymerases. (A) The sequence alignment of C-terminal domains (CTDs) and (B) N-terminal domains (NTDs) of yeast mitochondrial RNA polymerase (y-mtRNAP), T7 RNA polymerase, and human mitochondrial RNA polymerase (h-mtRNAP). The secondary structure of y-mtRNAP is shown over the aligned sequences. The regions covering thumb, palm, fingers, and the yeast-characteristic insert are marked as green, red, blue, and gray boxes. The pictures were generated using ESPript server (http://espript.ibcp.fr/ESPript/ESPript/). (C) A stereo view of y-mtRNAP PmlC showing the interaction of the NTD (green) of $y$-mtRNAP with the upstream DNA promoter (pink template and cyan non-template). The NTD of h-mtRNAP NTD (red, PDB Id. 6EQR) is superimposed. The aligned structural elements are labeled black and the non-aligned ones are labeled green for $y$ mtRNAP and red for h-mtRNAP. 

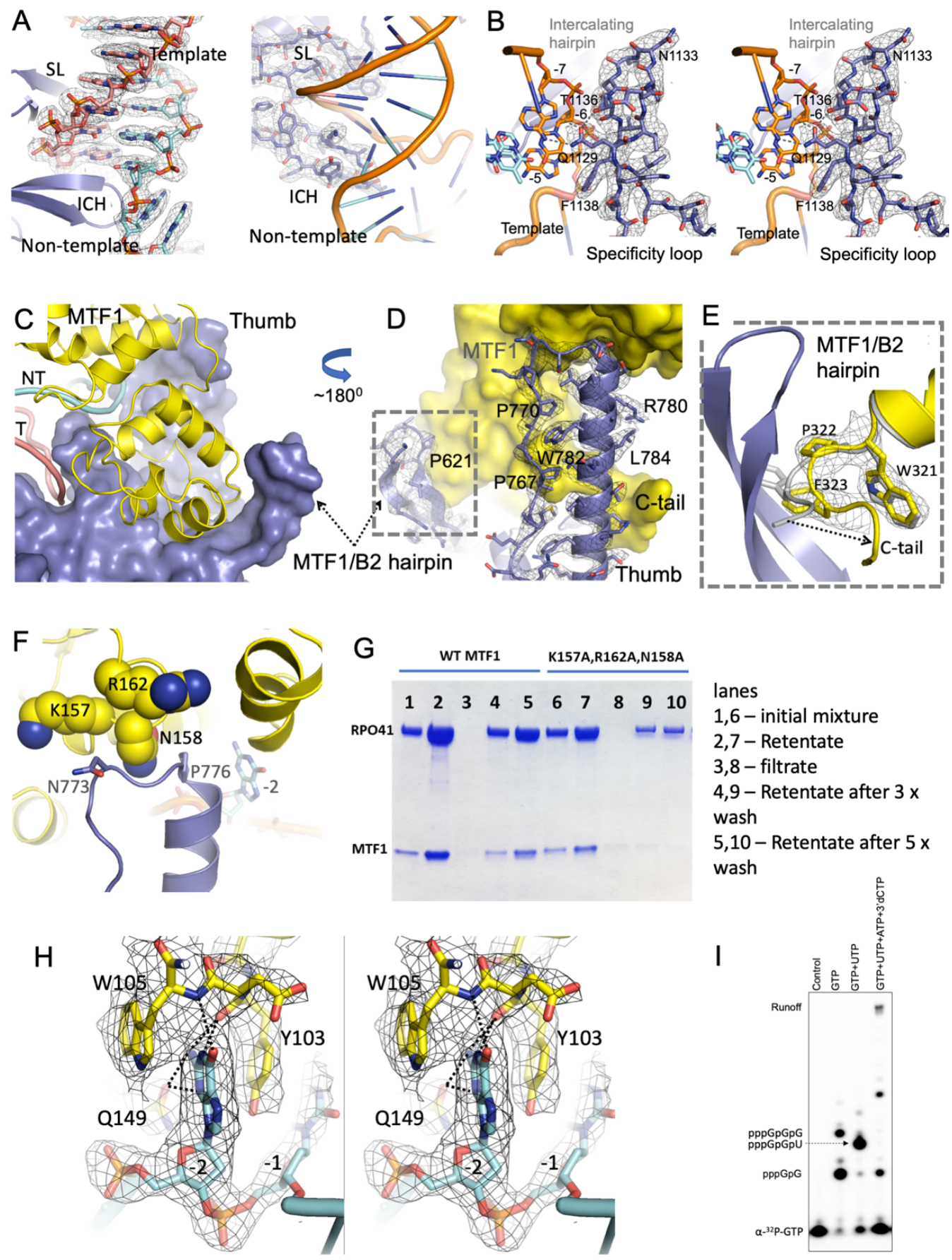

Figure S4. Structural and biochemical analysis of interactions among y-mtRNAP, MTF1, and DNA. (A) Density for DNA (left) and for intercalating hairpin (ICH) and specificity loop (SL) interacting with DNA (right) define their relative locations. (B) A stereo view showing the interactions of SL with the upstream DNA promoter; the template and non-template strands are in pink and cyan C-atoms representations, respectively. (C) Y-mtRNAP thumb, ICH, and MTF1supporting hairpin (613-632) place MTF1 (yellow ribbon) in y-mtRNAP PmIC. (D) The $3.1 \AA$ resolution density map defines thumb-domain interactions with MTF1 (yellow surface). (E) A 
zoomed view of the highlighted region in panel D shows the MTF1-supporting loop guiding the C-tail inward; the arrow shows the repositioning of C-tail base in MTF1-only structure (PDB Id. 1I4W; gray) to that in $\mathrm{y}$-mtRNAP IC. (F) MTF1 residues K157, N158, and R162 that interact with the tip of the thumb were mutated to alanine. (G) The triple alanine mutant MTF1 was investigated for complex formation with y-mtRNAP by an ultrafiltration assay. Lane 5 shows a stable y-mtRNAP:MTF1 complex after five washes whereas the mutant MTF1 is washed out, as shown in lane 10. $(\mathrm{H})$ Stereo view showing the aromatic ring stacking and a hydrogen bond network of -2 NT guanine base with MTF1; the view is flipped by $\sim 180^{\circ}$ compared to Figure $2 \mathrm{E}$. (I) Transcription initiation by $y$-mtRNAP IC on a pre-melted $15 S$ yeast mtDNA promoter that was used for structural study.

Image of the $24 \%$ polyacrylamide, $4 \mathrm{M}$ Urea denaturing gel shows runoff and abortive products from transcription reactions performed with $1 \mu \mathrm{M} y$-mtRNAP, $2 \mu \mathrm{M}$ MTF1 WT and $2 \mu \mathrm{M}$

promoter for $15 \mathrm{~min}$ in presence of the $100 \mu \mathrm{M}$ each of ATP, UTP, GTP, and 1.25 mM 3'-dCTP. Reactions were spiked with a small amount of $\alpha-{ }^{32} P-G T P$. Reactions with limited nucleotides (GTP or GTP and UTP) produced 2-mer and 3-mer markers. 

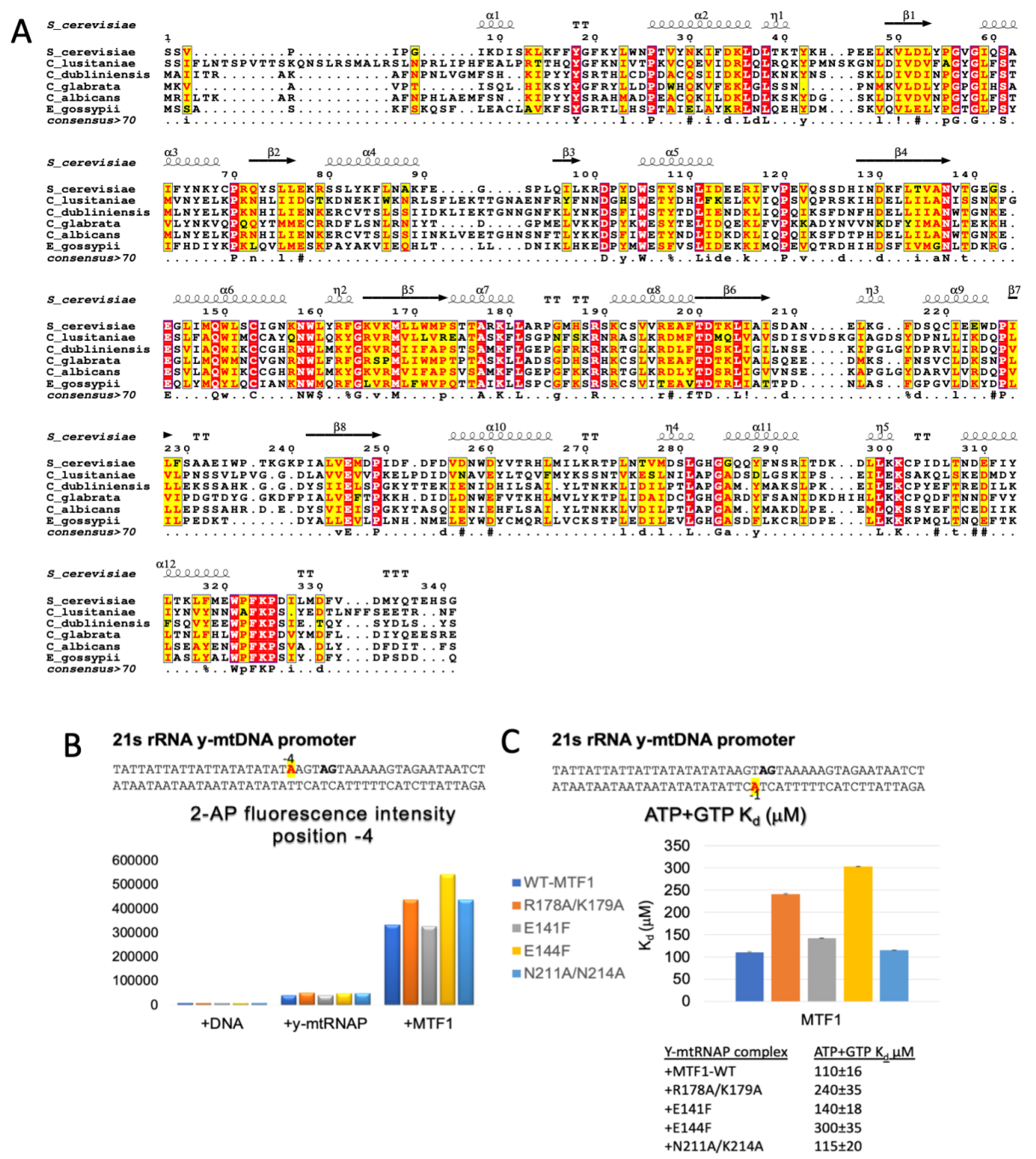

Figure S5. Amino acid sequence comparison of fungal MTF1 proteins and 2-AP promoter melting and initiating NTP binding studies of mutant MTF1. (A) Sequences of yeast (Saccharomyces cerevisiae) MTF1 is aligned with the MTF1 sequences of a wide range of fungal species Candida lusitaniae (C_lusitaniae), Candida dubliniensis (C_dubliniensis), Candida glabrata, (C_glabrata), Candida albicans (C_albicans), and Eremothecium gossypii (E_gossypii). The secondary structure of Saccharomyces cerevisiae MTF1 from 3.1 A PmIC complex is drawn over the aligned sequence. The picture was generated using ESPript server (http://espript.ibcp.fr/ESPript/ESPript/). (B) Promoter DNA was labeled with 2-aminopurine (2AP) at position -4 (in red) in the non-template strand. The fluorescence intensity (excitation at $315 \mathrm{~nm}$ and emission $380 \mathrm{~nm}$ ) of 2-AP labeled promoter $(200 \mathrm{nM})$ was recorded at $25^{\circ} \mathrm{C}$ before and after the addition of $400 \mathrm{nM} y-m$ tRNAP and $400 \mathrm{nM}$ WT or mutant MTF1 in succession. The increase in 2-AP fluorescence intensity is indicative of -4 position base unstacking. (C) The promoter DNA was labeled with $2 \mathrm{AP}$ at position -1 (in red) in the template strand. Increasing concentrations of initiating nucleotides (ATP+GTP) were added to a complex of 2AP promoter DNA (200 nM), ymtRNAP $(400 \mathrm{nM})$, and WT or mutant MTF1 $(400 \mathrm{nM})$ at $25^{\circ} \mathrm{C}$. Fluorescence emission at $380 \mathrm{~nm}$ after excitation at $315 \mathrm{~nm}$ was recorded. The titration data were fit to a hyperbolic equation to estimate the cumulative $\mathrm{K}_{\mathrm{d}}$ of the initiating nucleotides plotted and listed with standard deviations. 


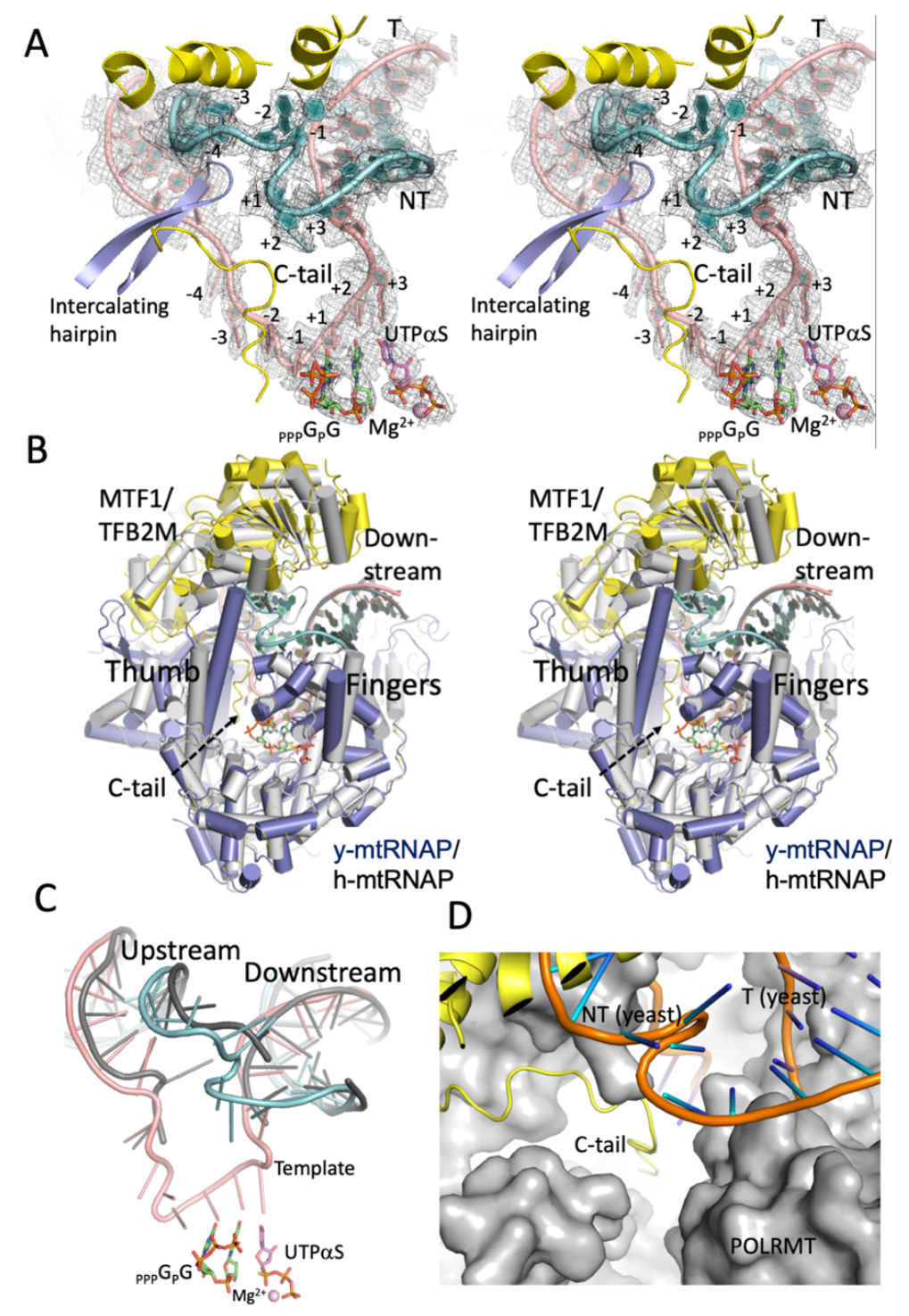

Figure S6. Comparison of y-mtRNAP IC and $h$-mtRNA IC structures. (A) A stereo view showing transcription bubble in y-mtRNAP IC. The cryo-EM density map at $3.7 \AA$ resolution helped building the DNA reliably. Most protein atoms are omitted for a clear view. The nontemplate (NT; cyan) and template (T; pink) nucleotides in the bubble are numbered. The pppGpG $(R N A)$ and UTP $\alpha S$ are in green and magenta $C$-atom representations, respectively $(A) A$ stereo view of the overlaid y-mtRNAP IC structure on h-mtRNAP IC (PDB ID. 6EQR) based on superposition of $y$-mtRNAP and $h$-mtRNAP shows a very similar architecture of mtRNAP ICs. The $\mathrm{h}-\mathrm{mtRNAP}$ IC components $\mathrm{h}-\mathrm{mtRNAP}$ and TFB2M are in gray and the promoter DNA in dark gray. The y-mtRNAP IC components y-mtRNAP, MTF1, non-template and template are in blue, yellow, cyan, and pink, respectively. (B) The tracks of the upstream and downstream DNA in both structures are well superimposed; the colors of DNA strands are same as in A. (C) Based on the above superposition, the C-tail of $y$-mtlC is positioned in the active-site cavity of $h$-mtRNAP IC suggesting that the TFB2M C-tail has an analogous role in h-mtlC to the role of MTF1 C-tail in ymIRNAP IC. 

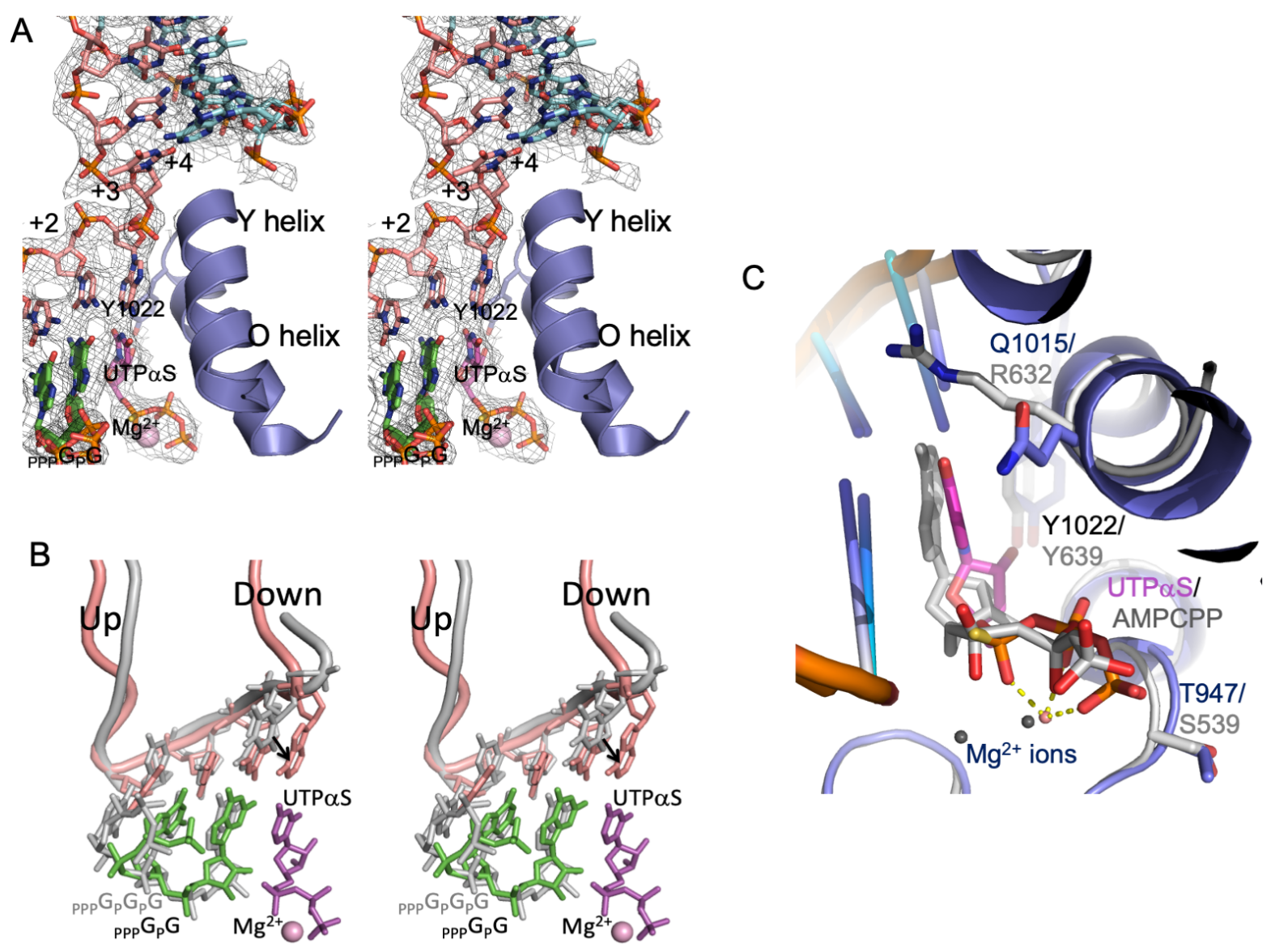

Figure S7. Positioning of the template, RNA and NTP at the active site region in y-mtRNAP IC structure. (A) A stereo view of the polymerase active-site region of $y$-mtIC structure showing the relative positioning of O-helix and $Y$-helix with respect to the template (pink), RNA (green) and UTP $\alpha S$ (magenta); $3.7 \AA$ cryo-EM map defines the locations and tracks of nucleic acid and UTP in the $y$-mtRNAP IC structure. (B) A stereo view showing the superimposed template (pink), pppGpG RNA (green) of $y$-mtRNAP IC on the template and pppGpGpG RNA of T7 RNAP IC (gray, PDB: 1QLN); the alignment is based on the superposition of $y$-mtRNAP and T7 RNAP. (C) Superposition of the NTP-binding sites of $y$-mtRNAP IC (blue protein, magenta UTP $\alpha$ S, cyan DNA, and pink $\mathrm{Mg}^{2+}$ ion) and T7 RNAP elongation complex (PDB Id. 1S76; gray). T7 RNAP structure has bound non-hydrolysable AMPCPP, which has a distorted active-site metal ion chelation. All NTP-binding pocket residues are conserved except Q1015/R632 and T947/S539 for mtRNAP/T7 RNAP. 